\title{
The nature of the interstellar medium of the starburst low-metallicity galaxy Haro 11: a multi-phase model of the infrared emission ${ }^{\star}$
}

D. Cormier ${ }^{1}$, V. Lebouteiller ${ }^{1}$, S. C. Madden ${ }^{1}$, N. Abel ${ }^{2}$, S. Hony ${ }^{1}$, F. Galliano ${ }^{1}$, M. Baes ${ }^{3}$, M. J. Barlow ${ }^{6}$, A. Cooray ${ }^{4}$, I. De Looze ${ }^{3}$, M. Galametz ${ }^{5}$, O. Ł. Karczewski ${ }^{6}$, T. J. Parkin ${ }^{7}$, A. Rémy ${ }^{1}$, M. Sauvage ${ }^{1}$, L. Spinoglio ${ }^{8}$, C. D. Wilson ${ }^{7}$, and R. $\mathrm{Wu}^{1}$

\author{
${ }^{1}$ Laboratoire AIM, CEA/DSM - CNRS - Université Paris Diderot, Irfu/Service d'Astrophysique, CEA Saclay, 91191 Gif-sur-Yvette, \\ France \\ e-mail: diane.cormier@cea.fr \\ 2 University of Cincinnati, Clermont College, Batavia, OH, 45103, USA \\ 3 Sterrenkundig Observatorium, Universiteit Gent, Krijgslaan 281 S9, 9000 Gent, Belgium \\ ${ }^{4}$ Department of Physics \& Astronomy, University of California, Irvine, CA 92697, USA \\ ${ }^{5}$ Institute of Astronomy, University of Cambridge, Madingley Road, Cambridge, CB3 OHA, UK \\ ${ }^{6}$ Department of Physics and Astronomy, University College London, Gower Street, London WC1E 6BT, UK \\ 7 Dept. of Physics \& Astronomy, McMaster University, Hamilton, Ontario, L8S 4M1, Canada \\ 8 Istituto di Fisica dello Spazio Interplanetario, INAF, via del Fosso del Cavaliere 100, 00133 Roma, Italy
}

Received 14 June 2012 / Accepted 28 August 2012

\begin{abstract}
Context. The low-metallicity interstellar medium (ISM) is profoundly different from that of normal systems, being clumpy with low dust abundance and little CO-traced molecular gas. Yet many dwarf galaxies in the nearby universe are actively forming stars. As the complex ISM phases are spatially mixed with each other, detailed modeling is needed to understand the gas emission and subsequent composition and structure of the ISM.

Aims. Our goal is to describe the multi-phase ISM of the infrared bright low-metallicity galaxy Haro 11, dissecting the photoionised and photodissociated gas components.

Methods. We present observations of the mid-infrared and far-infrared fine-structure cooling lines obtained with the Spitzer/IRS and Herschel/PACS spectrometers. We use the spectral synthesis code Cloudy to methodically model the ionised and neutral gas from which these lines originate.

Results. We find that the mid- and far-infrared lines account for $\sim 1 \%$ of the total infrared luminosity $L_{\mathrm{TIR}}$, acting as major coolants of the gas. Haro 11 is undergoing a phase of intense star formation, as traced by the brightest line, [O III] $88 \mu \mathrm{m}$, with $\left.L_{[\mathrm{O}} \mathrm{III}\right] / L_{\mathrm{TIR}} \sim 0.3 \%$, and high ratios of $[\mathrm{Ne} \mathrm{III}] /[\mathrm{Ne} \mathrm{II}]$ and $[\mathrm{S} \mathrm{IV}] /[\mathrm{S} \mathrm{III}]$. Due to their different origins, the observed lines require a multi-phase modeling comprising: a compact H II region, dense fragmented photodissociation regions (PDRs), a diffuse extended low-ionisation/neutral gas which has a volume filling factor of at least $90 \%$, and porous warm dust in proximity to the stellar source. For a more realistic picture of the ISM of Haro 11 we would need to model the clumpy source and gas structures. We combine these 4 model components to explain the emission of 17 spectral lines, investigate the global energy balance of the galaxy through its spectral energy distribution, and establish a phase mass inventory. While the ionic emission lines of Haro 11 essentially originate from the dense $\mathrm{H}$ II region component, a diffuse low-ionisation gas is needed to explain the [Ne II], [N II], and [C II] line intensities. The [O III] $88 \mu \mathrm{m}$ line intensity is not fully reproduced by our model, hinting towards the possible presence of yet another low-density high-ionisation medium. The [O I] emission is consistent with a dense PDR of low covering factor, and we find no evidence for an X-ray dominated component. The PDR component accounts for only $10 \%$ of the [C II] emission. Magnetic fields, known to be strong in star-forming regions, may dominate the pressure in the PDR. For example, for field strengths of the order of $100 \mu \mathrm{G}$, up to $50 \%$ of the [C II] emission may come from the PDR.
\end{abstract}

Key words. galaxies: ISM - galaxies: individual: Haro11 - ISM: lines and bands - ISM: structure - techniques: spectroscopic radiative transfer

\section{Introduction}

Star formation in the most pristine environments of the early universe is poorly understood. The closest analogs to chemically unevolved systems are the low-metallicity dwarf galaxies of our local universe. But even those well-studied nearby

\footnotetext{
* Herschel is an ESA space observatory with science instruments provided by European-led Principal Investigator consortia and with important participation from NASA.
}

galaxies continue to plague us with fundamental questions on what triggers and regulates star formation in metal-poor gasrich conditions. Certainly, from an observational point of view, at all wavelengths, local universe low-metallicity dwarf galaxies show dramatic differences compared to their more metal-rich counterparts (Kunth \& Östlin 2000, for a review), in the midinfrared (MIR), far-infrared (FIR), and submillimeter (submm) wavelength regimes. These include the dearth of polycyclic aromatic hydrocarbons (PAHs), prominent hot MIR-emitting dust, 
submm excess, and enhanced [C II] $157 \mu \mathrm{m} / \mathrm{CO}(1-0)$ ratios (e.g. Madden 2008; Madden et al. 2012).

What physical properties, local as well as global, within the dwarf galaxies are we witnessing with these observational signatures? How do we turn these signatures into a realistic view of star formation under early universe conditions? How do basic local parameters, such as radiation field, density, compactness, filling factors, metallicity, etc. figure in the picture we have of star-forming dwarf galaxies? Much of the ambiguity surrounding these questions is due to the lack of understanding the precise role of critical diagnostics and of spatial resolution in the most nearby objects, especially in the FIR/submm, resulting in a mixture of different interstellar medium (ISM) phases in the integrated view of galaxies.

Low-metallicity star-forming dwarf galaxies host young massive stellar clusters (e.g. Turner et al. 2000; Beck et al. 2000; Johnson \& Kobulnicky 2003) which produce ultraviolet (UV) photons. The low abundance and prevailing hard radiation field can explain most of the differences observed in dwarf galaxies in destroying large grains and PAHs, in favor of smaller dust grains. The UV photons also photodissociate molecules such as CO, which are unable to adequately self-shield, thus reducing the $\mathrm{CO}$ abundance in metal-poor gas (e.g. Wolfire et al. 2010; Narayanan et al. 2012). Thus UV photons play an important role in the heating and the chemistry of the ISM, controlling the structure of H II regions and photodissociation regions (PDRs). While the heating of the gas is mainly due to photoionisation and gas-grain collisions in the $\mathrm{H}$ II regions and to the photoelectric effect and cosmic rays in the neutral phase, the cooling is normally dominated by radiative de-excitation of fine-structure lines, readily observed in the MIR and FIR/submm. The behavior of these tracers and their relationship to the star formation properties, have been extensively studied to access physical parameters in terms of the gas temperature, density, and the radiation field (e.g. Malhotra et al. 2001; Hunter et al. 2001; Brauher et al. 2008). These sudies make use of photoionisation and PDR models at the scale of individual clouds (Kaufman et al. 1999; Wolfire et al. 1990; Ferland et al. 1998; Abel et al. 2005; Röllig et al. 2006; Tielens \& Hollenbach 1985).

The [C II] $157 \mu \mathrm{m}$ line is an important FIR diagnostic often put forward as a star formation tracer (Stacey et al. 2010; Boselli et al. 2002; de Looze et al. 2011). It has been observed in many different object types, including dense star-forming regions (Stacey et al. 1991), diffuse ionised and atomic regions (Madden et al. 1993), and high-redshift galaxies (Maiolino et al. 2009; Stacey et al. 2010). Since the [C II] line is well-correlated with the ${ }^{12} \mathrm{CO}(1-0)$ line and the FIR luminosity (e.g. Stacey et al. 1991; Pierini et al. 2003), it is generally considered that the $[\mathrm{C} \mathrm{II}$ ] line traces massive star formation within the PDR paradigm. [C II] is normally the brightest FIR cooling line, being much brighter than $\mathrm{CO}$, and carrying about 0.1 to $\sim 0.5 \%$ of the FIR luminosity in metal-rich star-forming galaxies. In lowmetallicity dwarf galaxies, where $\mathrm{CO}(1-0)$ is not easily detected because of photodissociation (e.g. Schruba et al. 2012; Leroy et al. 2011), [C II] can be as high as $1 \%$ of the $L_{\mathrm{FIR}}$ and often 5 to 10 times brighter relative to $\mathrm{CO}$ than in dustier starburst galaxies (e.g. Poglitsch et al. 1995; Madden et al. 1995; Madden 2000; Hunter et al. 2001; Cormier et al. 2010). The high $[\mathrm{C} \mathrm{II}] / \mathrm{CO}(1-0)$ ratio in low-metallicity systems may be alerting us to the fact that the morphology of their PDRs and H II regions and the relative filling factors of the various phases of dwarf galaxies differ from those of more metal-rich objects (e.g. Madden 2000; Kaufman et al. 2006; Röllig et al. 2006). Even in the nearest neighboring dwarf galaxies, the picture of star formation - and what controls this star formation - is enigmatic.

However, using [C II] alone can lead to ambiguous interpretation, particularly on the origin of the $\mathrm{C}^{+}$emission, which can arise from the ionised as well as neutral phases of the ISM. To employ the [C II] line as a valuable star formation tracer, it is necessary to model the different phases on galaxy-wide scales. The challenge rests on the unavoidable fact that on the scale of galaxies, aside from the closest dwarf galaxies such as the LMC (50 kpc, 1/2 $Z_{\odot}$ ), SMC (60 kpc, 1/5 $Z_{\odot}$ ), NGC 6822 (500 kpc, $\left.1 / 4 Z_{\odot}\right)$, or IC $10\left(800 \mathrm{kpc}, 1 / 3 Z_{\odot}\right)$, the phases of the ISM are mixed together in single telescope beams. When using a limited number of diagnostic lines, only a single galactic component can be modeled and average conditions derived, which is otherwise an ensemble of individual PDRs, ionised regions, etc. This requires modeling a comprehensive suite of tracers covering a range of critical densities and excitation potentials to characterise the dense H II regions, the diffuse HII regions, PDRs, etc. self-consistently. This approach allow us to grapple with degeneracies and ambiguities in the interpretation of these tracers. In this way, valuable observational diagnostics can be turned into a more accurate description of the multi-phase configuration of a full galaxy.

The Herschel Space Observatory (Pilbratt et al. 2010) has brought the opportunity to obtain a wide range of valuable FIR fine-structure diagnostics never observed before in dwarf galaxies. The Dwarf Galaxy Survey (DGS; P.I. Madden) is surveying the dust and gas of a wide range of low metallicity galaxies in the local universe (Cormier et al. 2010; Galametz et al. 2010; O’Halloran et al. 2010; Madden et al., in prep.; Rémy et al., in prep.) using PACS (Poglitsch et al. 2010) and SPIRE (Griffin \& et al. 2010). The brightest low-metallicity galaxy of the DGS observed with the PACS spectrometer is Haro 11, an infrared-luminous starburst galaxy with metallicity $Z=1 / 3 Z_{\odot}$. Haro 11 has the largest dataset obtained with PACS with observations of 7 FIR fine-structure lines: [C II] 157, [O III] 88, [O I] 63, [O I] 145, [N III] 57, [N II] 122, and [N II] $205 \mu \mathrm{m}$. It has also been observed with the IRS spectrograph onboard Spitzer. Unresolved in the IR, Haro 11 is an ideal candidate to conduct a study modeling an exhaustive set of gas tracers to retrieve information about the structure of the multi-phase lowmetallicity ISM.

In this paper, we propose a systematic approach to model the multi-phase ISM of Haro 11, focusing on the gas properties of the ionised and neutral ISM phases. We consider a set of 17 Herschel and Spitzer MIR and FIR ionic and atomic finestructure cooling lines which trace a wide range of environmental conditions, spanning excitation potentials from 8 to $55 \mathrm{eV}$. With the spectral synthesis code Cloudy (Ferland et al. 1998), we create a self-consistent model of the dominant ISM components from which these lines originate in order to understand the prevailing mechanisms and physical conditions. In Sect. 2, we present spectroscopic data from the Spitzer and Herschel observatories. Section 3 explains the modeling strategy and assumed initial parameters. Sections 4-6 report the model results and conditions of the ISM components. This study establishes an encompassing picture of the dominant ISM phases in Haro 11 and describes the mass budget of the various phases in our model (Sect. 7).

\subsection{Studies of Haro 11}

Several studies of Haro 11 have highlighted its peculiarity as a galaxy and challenged our understanding of star formation. 


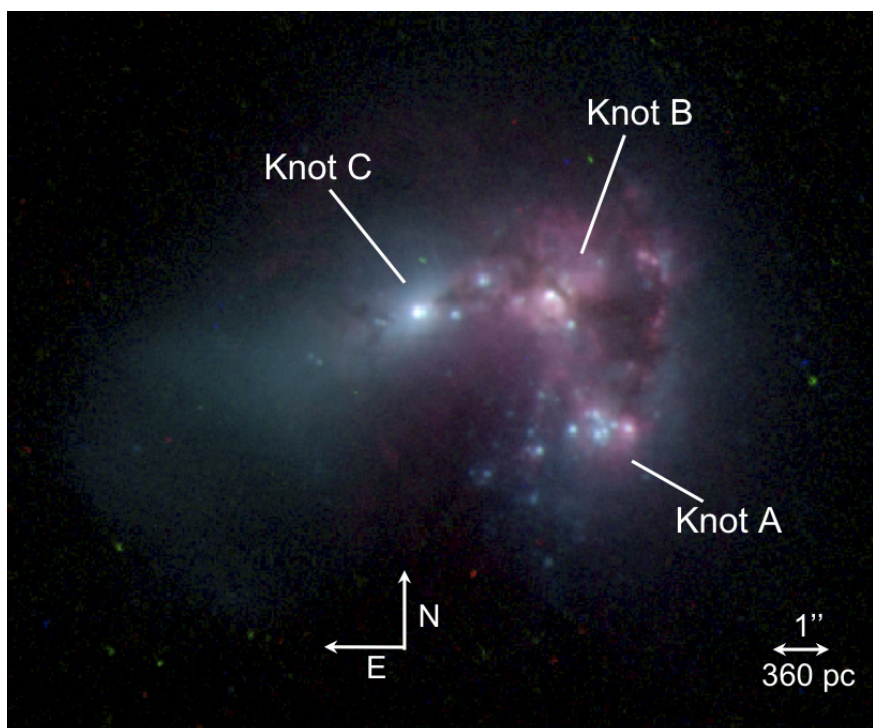

Fig. 1. HST/ACS 3-color image of Haro 11 with labels for the 3 starforming knots (blue: F435, green: F550, red: FR656N/H $\alpha$ ). Images were downloaded from the Hubble Legacy Archive. (This figure is available in color in electronic form.)

Haro 11 - also know as ESO 350-IG38 - is a blue compact dwarf (BCD) galaxy located at $84 \mathrm{Mpc}$, with $M_{B}=-20.3$, and metallicity $Z \sim 1 / 3 Z_{\odot}$ (Guseva et al. 2012). It is composed of 3 main star-forming regions, or knots, described in Vader et al. (1993) and Kunth et al. (2003), with a morphology presumably resulting from a merger event (Östlin et al. 2001). The starbursting nature of Haro 11 is visible in Fig. 1 with a dominant bright stellar component and dust lanes in front of knot B. These knots host hundreds of young star clusters and $\sim 60$ super star clusters (SSC) with ages between 1 and $100 \mathrm{Myr}$ peaking at $3 \mathrm{Myr}$, and constituting a total stellar mass of $10^{10} M_{\odot}$ (Adamo et al. 2010). An older stellar population is also present, primarily in knots A and $\mathrm{C}$, as seen in the red colors of the $V$ - $K$ bands, modeled in Micheva et al. (2010) by a metal-poor $(Z=0.001)$ stellar population of age around 14 Gyr with standard Salpeter IMF, indicating that the galaxy is not undergoing its first star formation event. Ly $\alpha$ emission (Kunth et al. 1998) and Lyman continuum leakage were investigated using UV and X-ray observations in Bergvall et al. (2006), Hayes et al. (2007), Grimes et al. (2007), and Leitet et al. (2011). In Leitet et al. (2011), they estimated the leakage to be $3 \%$. In particular, the discrepant location of $\mathrm{Ly} \alpha$ and $\mathrm{H} \alpha$ emission, originating in the ionised gas surrounding the star-forming regions, may be explained by a diffuse ionised ISM in the halo of the galaxy with internal resonant scattering at the surface of clumpy dense neutral clouds (Kunth et al. 2003; Hayes et al. 2007). These properties have resulted in its popularity as a local analogue of the high-redshift Lyman-break galaxies (Overzier et al. 2008), and therefore a must-study case to link nearby star formation to the distant universe and galaxy evolution.

In the IR, Haro 11 is extremely bright compared to other nearby dwarf galaxies. IRAS measurements yielded a FIR luminosity $L_{\mathrm{FIR}}$ of $1 \times 10^{11} L_{\odot}$ (Sanders et al. 2003a) from 40 to $400 \mu \mathrm{m}$ using the Lonsdale et al. (1985) prescription. Its spectral energy distribution (SED) shows a peculiar behavior. It peaks around $40 \mu \mathrm{m}$, indicating the prevalence of warm dust, and shows an excess of emission in the submm, as unveiled by APEX/LABOCA and Herschel, which is often seen in low-metallicity starbursting dwarf galaxies (Galliano et al. 2003, 2005; Galametz et al. 2009, 2011; Rémy et al., in prep.). The origin of the excess is not well determined and several
Table 1. General properties of Haro 11.

\begin{tabular}{lcl}
\hline \hline Quantity & Value & Reference \\
\hline RA (J2000) & $00 \mathrm{~h} 36^{\prime} 52.5^{\prime \prime}$ & $\mathrm{NED}^{a}$ \\
Dec (J2000) & $-33 \circ 33^{\prime} 19^{\prime \prime}$ & $\mathrm{NED}^{\prime}$ \\
Distance & $84 \mathrm{Mpc}$ & NED $^{b}$ \\
12+log(O/H) & 8.2 & Guseva et al. (2012) \\
Optical size & $0.4^{\prime} \times 0.5^{\prime}$ & NED \\
$L_{\mathrm{TIR}}{ }^{c}$ & $1.4 \times 10^{11} L_{\odot}$ & Galametz et al. (2009) \\
$L_{\mathrm{FUV}}$ & $2 \times 10^{10} L_{\odot}$ & Grimes et al. (2007) \\
$L_{\mathrm{X}-\text { rays }}$ & $4.4 \times 10^{7} L_{\odot}$ & Grimes et al. (2007) \\
$L_{\mathrm{Ly} \alpha}$ & $1.9 \times 10^{8} L_{\odot}$ & Hayes et al. (2007) \\
$L_{\mathrm{H} \alpha}$ & $8 \times 10^{8} L_{\odot}$ & Östlin et al. (1999) \\
\hline
\end{tabular}

Notes. ${ }^{(a)}$ NASA/IPAC Extragalactic Database. ${ }^{(b)}$ This distance is derived with a Hubble constant $H_{0}=73 \mathrm{~km} \mathrm{~s}^{-1} \mathrm{Mpc}^{-1}$. ${ }^{(c)} L_{\mathrm{TIR}}$ is the integrated SED from 3 to $1100 \mu \mathrm{m}$. All luminosities are scaled to the distance chosen in this paper of $84 \mathrm{Mpc}$.

hypotheses described in Galametz et al. (2011), including a change of dust emissivity, spinning dust grains, or a cold dust component, are invoked in current dust models to explain the submm excess. Galametz et al. (2009) present a detailed dust modeling of Haro 11 in which they derive a total dust mass of $6 \times 10^{6} M_{\odot}$ if the submm excess is ignored, and of $2 \times 10^{7} M_{\odot}$ if the submm excess is interpreted as cold dust. Assuming the excess as cold dust $(T \sim 10 \mathrm{~K})$ is however difficult to reconcile with the observed dust and gas mass budgets, and the expected lower dust-to-gas mass ratios (DGR; Galametz et al. 2011). Integrating the SED from 3 to $1100 \mu \mathrm{m}$, the total IR luminosity $L_{\mathrm{TIR}}$ is $1.4 \times 10^{11} L_{\odot}$ (Galametz et al. 2009). We will use this value of $L_{\mathrm{TIR}}$ throughout this paper.

Spectroscopic observations show bright lines in the FIR with ISO/LWS, while both the H I 21-cm line, and the CO $(J=1-0)$ line, the most common tracer of molecular gas in galaxies, are undetected (Bergvall et al. 2000). A detailed study of the neutral gas of the ISM of Haro 11 by Bergvall et al. (2000) investigated the mass budget of the different ISM phases. They establish masses for each phase: $M(\mathrm{HI})<10^{8} M_{\odot}$, with an upper limit on the atomic H I; M(PDR) of $2 \times 10^{8} M_{\odot}$ using the LWS [C II] observations, the PDR mass being larger than the HI mass; and $M\left(\mathrm{H}_{2}\right) \sim 10^{8} M_{\odot}$ for the molecular gas, using both a scaling of the PDR mass and the $\mathrm{CO}$ upper limit converted to $\mathrm{H}_{2}$ column density through the $\mathrm{CO} / \mathrm{H}_{2}$ conversion factor $\left(X_{\mathrm{CO}}\right)$. They used a Galactic value for $X_{\mathrm{CO}}$ (Strong et al. 1988): $X_{\mathrm{CO} \text {,gal }}=2.3 \times 10^{20} \mathrm{~cm}^{-2}\left(\mathrm{~K} \mathrm{~km} \mathrm{~s}^{-1}\right)^{-1}$. Therefore they describe the ISM structure as: very extended PDRs, in which the H I mass resides; a small fraction of the total ISM gas is left in atomic form; and the bulk of the gas, that is normally in neutral state, is ionised by the starburst. Bergvall \& Östlin (2002) derive $M(\mathrm{H}$ II $) \sim 10^{9} M_{\odot}$ from $\mathrm{H} \alpha$ observations for the ionised gas. These masses are lower than the total stellar mass of $10^{10} M_{\odot}$ derived in Adamo et al. (2010).

However, important questions remain, particularly on the state of the ionised, neutral atomic, and molecular gas. While evidence for young massive stellar populations and SSCs exist from the optical and IR point of view, evidence for any substantial reservoir of neutral gas, in the form of either atomic or molecular, is non-existent to date. Where is the fuel for the vigorous star formation observed in Haro 11? What is the state of the gas reservoirs in Haro 11, residing in the ionised, neutral, and molecular form? This study, using a wide variety of tracers of neutral and ionised gas, aims for a more complete description of the enigmatic nature of the ISM of Haro 11. 


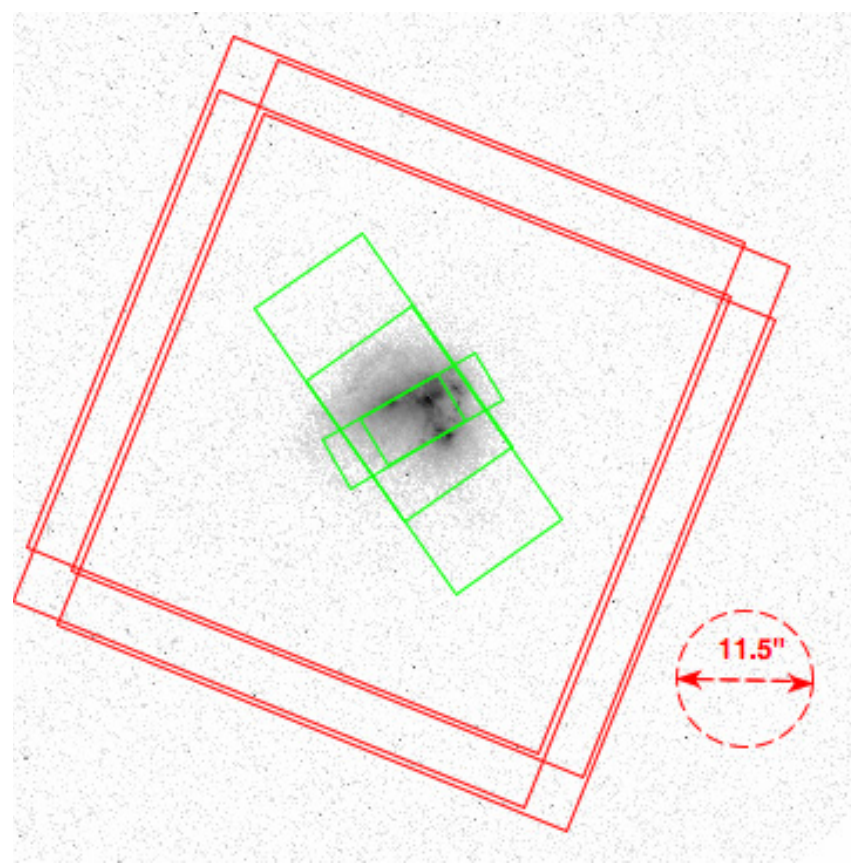

Fig. 2. $\mathrm{H} \alpha$ image of Haro 11 from the Hubble Legacy Archive. The Spitzer/IRS high-resolution slits are overlaid in green and the Herschel/PACS array in red. The PACS beamsize at $150 \mu \mathrm{m}$ is $11.5^{\prime \prime}$. The IRS low-resolution slits are not shown. (This figure is available in color in electronic form.)

\section{Observations}

\subsection{Spitzer data}

Haro 11 was observed with the Infrared Spectrograph (IRS; Houck et al. 2004) on board the Spitzer Space Telescope (Werner et al. 2004), on the 17th of July 2004 (P.I.: J. R. Houck). The 4 modules were used, i.e., the 2 high-resolution modules: Short-High SH $(\approx 10-20 \mu \mathrm{m})$ and Long-High LH $(\approx 19-37 \mu \mathrm{m})$ with $R \sim 600$, and the 2 low-resolution modules: Short-Low SL $(\approx 5-14 \mu \mathrm{m})$ and Long-Low LL $(\approx 14-37 \mu \mathrm{m})$ with $R \sim 60-120$. Observations were done in staring mode (AORKey 9007104). Figure 2 shows the slits of the high-resolution module on the sky.

The low-resolution spectra were retrieved from the CASSIS Atlas $^{1}$ (Lebouteiller et al. 2011), with a tapered spatial window that scales with wavelength to account for the broader PSF at longer wavelengths. The high-resolution spectra were obtained with SMART v8.2.2 (Lebouteiller et al. 2010; Higdon et al. 2004) from the full-slit extraction of SH and LH and assuming a point-like source calibration. The SL and SH spectra were stitched to LL and LH by applying the same factor 1.13, which is due to the fact that the source is slightly extended for the SL and $\mathrm{SH}$ apertures (3.7" and $4.7^{\prime \prime}$ height respectively), while it is point-like for the LL and LH apertures $\left(\approx 11^{\prime \prime}\right)$. Another factor of 1.15 was applied to the low-resolution spectra to match the continuum of the high-resolution. The final rest-frame spectrum is displayed in Fig. 3 (top), with a zoom on individual spectral lines (bottom). With a spectral resolution $\geq 500 \mathrm{~km} \mathrm{~s}^{-1}$, the lines are not spectrally resolved.

Line fitting and flux measurements were also done in SMART. A Gaussian and second order polynomial function

\footnotetext{
1 The Cornell Atlas of Spitzer/IRS Sources (CASSIS) is a product of the Infrared Science Center at Cornell University, supported by NASA and JPL.
}

were fitted to the line and continuum. For total uncertainties, we added $10 \%$ of the line flux due to calibration uncertainties. The resulting fluxes and uncertainties of the most prominent lines are listed in Table 2. The fluxes agree within the uncertainties to those of $\mathrm{Wu}$ et al. (2006). The [Ne III] line at $36.0 \mu \mathrm{m}$ was not observed as it falls out of the IRS spectral range.

\subsection{Herschel data}

Haro 11 was observed with the PACS spectrometer (Poglitsch et al. 2010) on the 27th of June 2010, OD 409 of the Herschel mission (Pilbratt et al. 2010) as part of the Guaranteed Time Key Program, the Dwarf Galaxy Survey (P.I.: S. C. Madden). The PACS array is composed of $5 \times 5$ spatial pixels each 9.4" square, covering a total field-of-view of 47". Haro 11 was mapped with $2 \times 2$ rasters separated by $4.5^{\prime \prime}$ in the 7 following fine-structure spectral lines: [C II] $157 \mu \mathrm{m}$, [O III] $88 \mu \mathrm{m}$, [O I] $145 \mu \mathrm{m}$ (OBSID 1342199236), [O I] $63 \mu \mathrm{m}$, [N III] $57 \mu \mathrm{m}$, [N II] $122 \mu \mathrm{m}$ (OBSID 1342199237), and [N II] $205 \mu \mathrm{m}$ (OBSID 1342199238), for a total of $7.2 \mathrm{~h}$. The [N II] $205 \mu \mathrm{m}$ line was also re-observed on OD 942 for $1.6 \mathrm{~h}$ with a single pointing (OBSID 1342234063). The observations were done in chop-nod mode with a chop throw of $6^{\prime}$ off the source. The chopped position is free of emission. The beam size is $9.5^{\prime \prime}$ at $60 \mu \mathrm{m}$, and $11.5^{\prime \prime}$ at $150 \mu \mathrm{m}$ (diffraction limited above $\sim 100 \mu \mathrm{m})$. The spectral resolution is $\sim 90 \mathrm{~km} \mathrm{~s}^{-1}$ at $60 \mu \mathrm{m}$, $125 \mathrm{~km} \mathrm{~s}^{-1}$ at $90 \mu \mathrm{m}, 295 \mathrm{~km} \mathrm{~s}^{-1}$ at $120 \mu \mathrm{m}$, and $250 \mathrm{~km} \mathrm{~s}^{-1}$ at $150 \mu \mathrm{m}$ (PACS Observer's Manual 20112).

The data were reduced with the Herschel Interactive Processing Environment (HIPE) v7.0.1935 (Ott 2010). We used standard scripts of the PACS spectrometer pipeline. The line fitting and map making were done using the software PACSman (Lebouteiller et al. 2012). The signal from each spatial position of the PACS array is fitted with a second order polynomial plus Gaussian for the baseline and line. For total uncertainties, we add $30 \%$ of the line flux due to calibration uncertainties (Poglitsch et al. 2010). The rasters are combined by drizzling to produce final reduced maps with pixel size of $3^{\prime \prime}$.

With Herschel, all of the lines are well detected, at least in the central pixel of the PACS array, except the [N II] $205 \mu \mathrm{m}$ line. The [N II] $205 \mu \mathrm{m}$ line is located at the edge of the first grating order of the spectrometer and affected by spectral leakage, due to overlapping of the grating orders. It is contaminated by signal from the second grating order around $100 \mu \mathrm{m}$, and is therefore difficult to observe and to calibrate. The line profiles are displayed in Fig. 4. The line centers range between -30 and $+70 \mathrm{~km} \mathrm{~s}^{-1}$ within the maps of all observed lines, and indicate a rotation around the north-south axis of both the ionised and neutral gas components, slightly tilted compared to the velocity structure of $\mathrm{H} \alpha$ analysed in Östlin et al. (1999). All lines display broad profiles, with a broadening up to $300 \mathrm{~km} \mathrm{~s}^{-1}$, which suggests that both the neutral and ionised gas are coming from several components with different velocities. However, with spectral resolution $>100 \mathrm{~km} \mathrm{~s}^{-1}$, the lines are well fitted by a single Gaussian and we cannot separate the different velocity components in the spectra. This suggested complex velocity structure is corroborated by studies of optical lines that show broad profiles as well (Kunth et al. 1998; Östlin et al. 1999), and where the neutral gas is shifted compared to the ionised gas, expanding over few kpcs (Kunth et al. 2003). FUSE spectra in Grimes et al. (2007) show absorption lines of the ISM with $F W H M 300 \mathrm{~km} \mathrm{~s}^{-1}$ and blueshifted by $80 \mathrm{~km} \mathrm{~s}^{-1}$. The higher

\footnotetext{
2 Available at http://herschel.esac.esa.int/Docs/PACS/ html/pacs_om.html
} 
D. Cormier et al.: Modeling the ISM of Haro 11
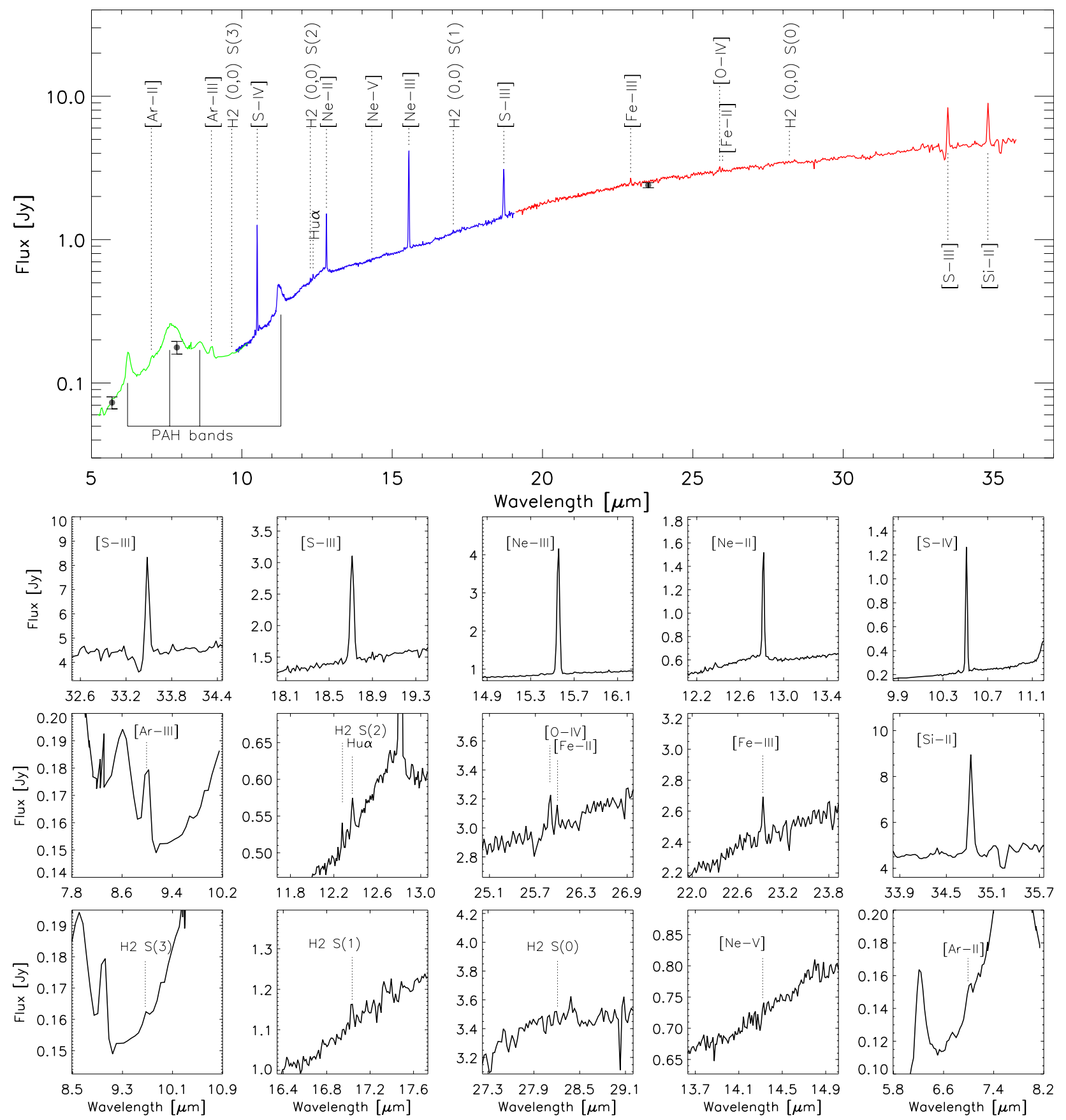

Fig. 3. Top: Spitzer/IRS spectrum of Haro 11 in the rest wavelength frame $(z=0.0206)$, SL in green, SH in blue, and LH in red. Emission lines are labeled on top. The IRAC and MIPS photometry is also overlaid (fluxes in Table 3). Bottom: zoom on the individual spectral lines. Fluxes and upper limits are reported in Table 2. (This figure is available in color in electronic form.)

ionisation lines can be stronger in galactic outflows (Contursi et al. 2012).

The [C II] line shows a marginally extended emission at $157 \mu \mathrm{m}$, peaking at a signal-to-noise ratio of 50 in the center and dropping below detection at the edge of the map. The emission of the other lines is rather compact. The shape of the PACS spectrometer beam is not known well enough to measure an accurate source size if the source were slightly extended. Hence we cannot recover spatial information. To derive total line intensities, we integrated the fluxes over a circular aperture of diameter $30^{\prime \prime}$ to encompass all the emission. The line intensities are listed in Table 2. Observations of the [O III], [C II], and [O I] 63 and $145 \mu \mathrm{m}$ lines were also performed with the LWS instrument onboard ISO (Bergvall et al. 2000;
Brauher et al. 2008; Vasta et al. 2010). The PACS intensities of the [O I] $63 \mu \mathrm{m}$ and [O III] lines agree within $40 \%$ with the LWS observations, although they are found to be lower with PACS. This may be attributed to a beam effect, the LWS beam size being $\sim 80^{\prime \prime}$. However, we find that the $[\mathrm{C} \mathrm{II}]$ intensity is two times brighter with PACS compared to LWS. To understand this discrepancy, we have reduced the PACS data with different flat-field corrections, as well as with background normalisation (PACS Data Reduction Guide). All these methods give the same final [C II] flux as quoted in Table 2 within 10\%, ruling out a miscalibration from PACS. Therefore we attribute the difference between the PACS and LWS fluxes to a calibration problem from the LWS instrument. 

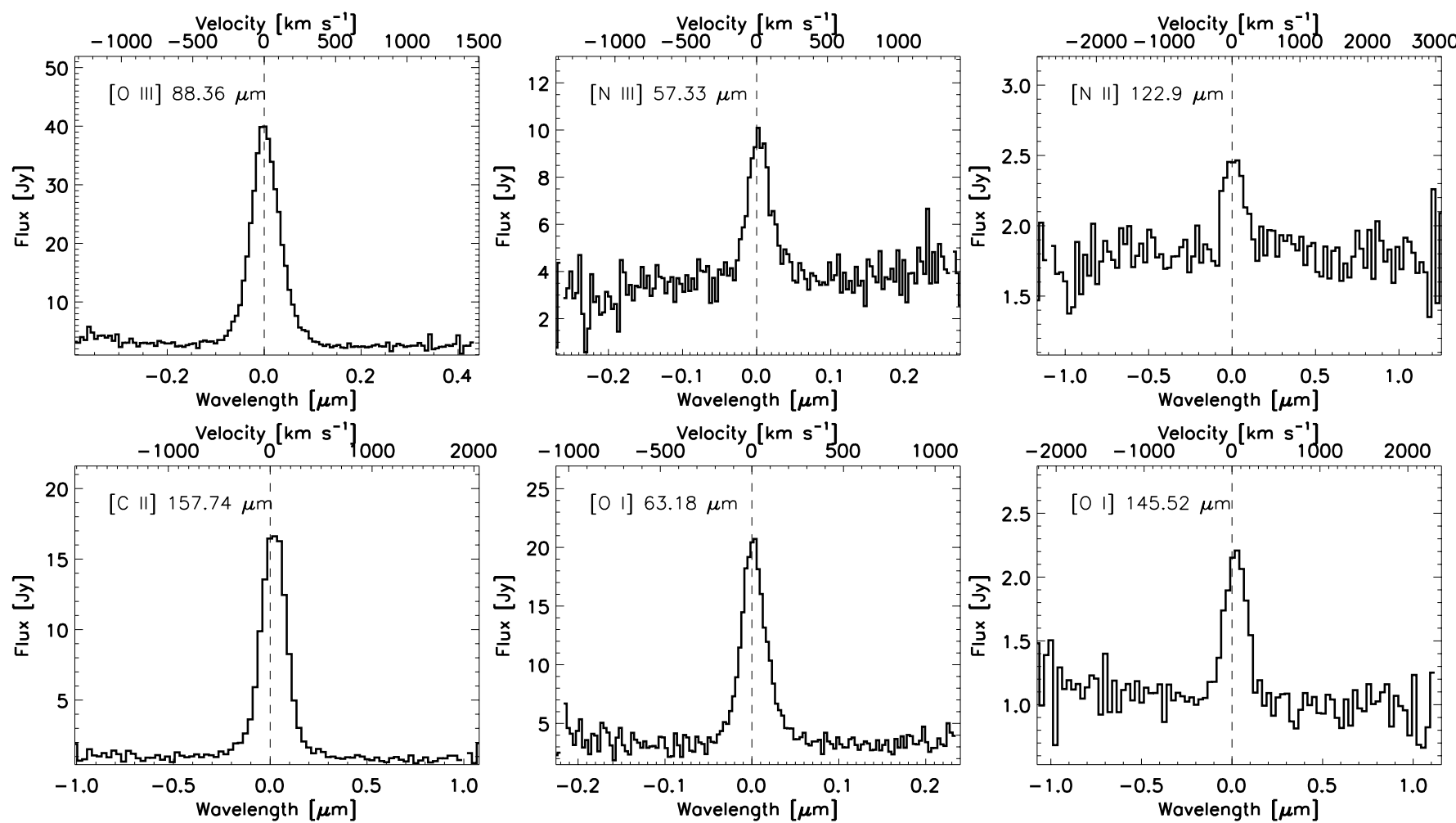

Fig. 4. Herschel/PACS line profiles from an individual spatial pixel (9.4") centered on the galaxy.

Table 2. Spitzer and Herschel line fluxes.

\begin{tabular}{|c|c|c|c|c|c|c|}
\hline Line & $\begin{array}{l}\text { Wavelength } \\
\qquad(\mu \mathrm{m})\end{array}$ & $\begin{array}{c}\text { Flux }^{a} \\
\left(10^{-16} \mathrm{~W} \mathrm{~m}^{-2}\right)\end{array}$ & $\begin{array}{c}\text { Uncertainty } \\
\left(10^{-16} \mathrm{~W} \mathrm{~m}^{-2}\right)\end{array}$ & $\begin{array}{l}\text { Excit. potential }^{b} \\
(\mathrm{eV})\end{array}$ & $\begin{array}{l}\text { Excit. temperature }^{c} \\
(\mathrm{~K})\end{array}$ & $\begin{array}{l}\text { Critical density }{ }^{d} \\
\left(\mathrm{~cm}^{-3}\right)\end{array}$ \\
\hline \multicolumn{7}{|l|}{ Spitzer/IRS } \\
\hline [S IV] & 10.51 & 5.37 & 0.66 & 34.79 & 1369 & $5 \times 10^{4}[\mathrm{e}]$ \\
\hline$[\mathrm{Ne} \mathrm{II}]$ & 12.81 & 3.53 & 0.43 & 21.56 & 1123 & $7 \times 10^{5}[\mathrm{e}]$ \\
\hline$[\mathrm{Ne}$ III] & 15.56 & 12.3 & 1.5 & 40.96 & 925 & $3 \times 10^{5}[\mathrm{e}]$ \\
\hline$[\mathrm{S}$ III $]$ & 18.71 & 5.79 & 0.72 & 23.34 & 769 & $2 \times 10^{4}[\mathrm{e}]$ \\
\hline [S III] & 33.48 & 5.85 & 0.69 & " & 430 & $7 \times 10^{3}[\mathrm{e}]$ \\
\hline [Si II $]$ & 34.82 & 6.21 & 0.75 & 8.15 & 413 & $3 \times 10^{5}[\mathrm{H}], 1 \times 10^{3}[\mathrm{e}]$ \\
\hline Humphreys $\alpha$ & 12.37 & 0.208 & 0.045 & 13.60 & 1163 & - \\
\hline $\mathrm{H}_{2}(0,0) \mathrm{S}(0)$ & 28.22 & $<0.62$ & - & 4.48 & 510 & $7 \times 10^{2}[\mathrm{H}]$ \\
\hline $\mathrm{H} 2(0,0) \mathrm{S}(1)$ & 17.03 & 0.168 & 0.055 & " & 1015 & $2 \times 10^{4}[\mathrm{H}]$ \\
\hline $\mathrm{H} 2(0,0) \mathrm{S}(2)$ & 12.28 & 0.101 & 0.013 & " & 1682 & $2 \times 10^{5}[\mathrm{H}]$ \\
\hline $\mathrm{H} 2(0,0) \mathrm{S}(3)$ & 9.66 & 0.121 & 0.018 & " & 2504 & $9 \times 10^{5}[\mathrm{H}]$ \\
\hline$[\mathrm{Fe} \mathrm{III}]$ & 22.93 & 0.54 & 0.20 & 16.19 & 627 & $1 \times 10^{5}[\mathrm{e}]$ \\
\hline [O IV $]$ & 25.89 & 0.49 & 0.18 & 54.94 & 555 & $1 \times 10^{4}[\mathrm{e}]$ \\
\hline$[\mathrm{Fe} \mathrm{II}]$ & 25.99 & $<0.25$ & - & 7.90 & 554 & $2 \times 10^{6}[\mathrm{H}], 1 \times 10^{4}[\mathrm{e}]$ \\
\hline [Ar III] & 8.99 & 0.99 & 0.33 & 27.63 & 2060 & $3 \times 10^{5}[\mathrm{e}]$ \\
\hline$[\mathrm{Ar}$ II $]$ & 6.99 & 0.60 & 0.15 & 15.76 & 1600 & $4 \times 10^{5}[\mathrm{e}]$ \\
\hline$[\mathrm{Ne} \mathrm{V}]$ & 14.32 & $<0.080$ & - & 97.12 & 592 & $3 \times 10^{4}[\mathrm{e}]$ \\
\hline \multicolumn{7}{|l|}{ Herschel/PACS } \\
\hline$[\mathrm{C} \mathrm{II}]$ & 157.74 & 7.4 & 2.2 & 11.26 & 91 & $3 \times 10^{3}[\mathrm{H}], 50[\mathrm{e}]$ \\
\hline [O III] & 88.36 & 18.0 & 5.4 & 35.12 & 163 & $5 \times 10^{2}[\mathrm{e}]$ \\
\hline$[\mathrm{O} \mathrm{I}]$ & 63.18 & 6.7 & 2.0 & - & 228 & $5 \times 10^{5}[\mathrm{H}]$ \\
\hline$[\mathrm{O} \mathrm{I}]$ & 145.52 & 0.56 & 0.17 & - & 99 & $1 \times 10^{5}[\mathrm{H}]$ \\
\hline$[\mathrm{N}$ III] & 57.32 & 2.50 & 0.78 & 29.60 & 251 & $3 \times 10^{3}[\mathrm{e}]$ \\
\hline$[\mathrm{N}$ II $]$ & 121.9 & 0.31 & 0.10 & 14.53 & 118 & $3 \times 10^{2}[\mathrm{e}]$ \\
\hline$[\mathrm{N}$ II $]$ & 205.18 & $<0.29$ & - & " & 70 & $45[\mathrm{e}]$ \\
\hline
\end{tabular}

Notes. The ISO/LWS fluxes can be found in Bergvall et al. (2000), Brauher et al. (2008), and Vasta et al. (2010). ${ }^{(a)}$ Upper limits are given as the 3- $\sigma$ uncertainty from the line fit. ${ }^{(b)}$ Excitation potential. Energy to create an ion. For $\mathrm{H}_{2}$, it is the energy to photodissociate the molecule. ${ }^{(c)}$ Excitation temperature. Temperature $T=\Delta E / k$ required to populate the transition level. ${ }^{(d)}$ Critical density $n_{\text {crit }}$ noted [H] for collisions with hydrogen atoms ( $T=100 \mathrm{~K}$ and $T=300 \mathrm{~K}$ for the $\mathrm{H}_{2}$ lines), [e] with electrons ( $T=10000 \mathrm{~K}$ ). Values are from Malhotra et al. (2001), Giveon et al. (2002), and Kaufman et al. (2006). 


\subsection{Spectral line description}

The IRS and PACS spectra probe the ionised and neutral media. The IRS spectrum shows a wealth of MIR fine-structure lines, the brightest being (in order) [Ne III] $15.6 \mu \mathrm{m}$, [Si II] $34.8 \mu \mathrm{m}$, [S III] 18.7 and $33.5 \mu \mathrm{m}$, [S IV] $10.5 \mu \mathrm{m}$, and [Ne II] $12.8 \mu \mathrm{m}$. PAH features at 6.2, 7.7, 8.6, 11.2, and $12.8 \mu \mathrm{m}$ are also present. The PACS spectrum also shows bright FIR lines, especially [O III], [C II], [O I] $63 \mu \mathrm{m}$, and [N III]. The [O III] line is the brightest of all, with $[\mathrm{O} \mathrm{III}] /[\mathrm{C} \mathrm{II}]=2.4$. While $[\mathrm{C} \mathrm{II}]$ is the brightest FIR fine-structure line in normal and dusty starburst galaxies (Brauher et al. 2008), we find that this is often not the case in low-metallicity dwarf galaxies (Hunter et al. 2001; Cormier et al. 2010; Madden et al., in prep.; Cormier et al., in prep.).

The young star-forming nature of this galaxy also agrees with intense [Ne III] $(41.0 \mathrm{eV}),[\mathrm{O} \mathrm{III}](35.1 \mathrm{eV}),\left[\begin{array}{ll}\mathrm{S} & \mathrm{IV}\end{array}\right]$ $(34.8 \mathrm{eV})$, and [N III] $(29.6 \mathrm{eV})$ lines observed, arising from relatively highly ionised regions. [O IV] $(54.9 \mathrm{eV})$ at $25.9 \mu \mathrm{m}$, which requires a relatively hard radiation field (Sect. 6.3), is also detected. The $[\mathrm{Ne} \mathrm{III}] /[\mathrm{Ne} \mathrm{II}]$ ratio is $3.5,[\mathrm{~S} \mathrm{IV}] /[\mathrm{S} \mathrm{III}]$ is 0.9 , and $[\mathrm{N} \mathrm{III]}] /[\mathrm{N} \mathrm{II}]$ is 8 . These ratios are indicative of a hard radiation field typical of BCDs (Thornley et al. 2000; Madden et al. 2006). While [C II] can come from both the diffuse ionised phase and the neutral phase, $[\mathrm{O} \mathrm{I}]$ traces only the neutral phase. The [O I] $63 \mu \mathrm{m} /[\mathrm{C}$ II $]$ ratio is 0.9 , similar to that found in other dwarf galaxies and dustier galaxies (Hunter et al. 2001; Brauher et al. 2008). Table 2 lists the ionisation energies of the observed lines, as well as the excitation temperatures and critical densities $\left(n_{\text {crit }}\right)$ of the transitions with collisions with $\mathrm{H}$ and/or $\mathrm{e}^{-}$.

Comparing line luminosities to the TIR luminosity, we find $L_{\text {[C II] }} / L_{\mathrm{TIR}}=0.1 \%, L_{[\mathrm{O} \text { III] }} / L_{\mathrm{TIR}}=0.3 \%$, and $L_{[\mathrm{O} \text { I] }} / L_{\mathrm{TIR}}=0.1 \%$. Altogether, the FIR lines represent $0.6 \%$ of the TIR luminosity; and when adding the MIR lines, they represent $1.2 \%$ of $L_{\mathrm{TIR}}$. Together these lines are responsible for most of the gas cooling of the ISM. Such line-to-TIR ratios are typical of star-forming galaxies (Malhotra et al. 2001; Brauher et al. 2008) ${ }^{3}$. Similar $L_{\text {[C II] }} / L_{\mathrm{TIR}}$ ratios are found in several high-redshift galaxies (Maiolino et al. 2009; Stacey et al. 2010). Croxall et al. (2012) find $L_{[\mathrm{C} \text { II] }} / L_{\mathrm{TIR}} \sim 0.5 \%$ and $L_{[\mathrm{O} \text { I] }} / L_{\mathrm{TIR}} \sim 0.1 \%$ in spatially resolved regions of the nearby spiral galaxy NGC 4559. There is observational evidence that $L_{[\mathrm{C} \text { II }} / L_{\mathrm{TIR}}$ tends to decrease in galaxies with warmer FIR-color, $f_{v}(60 \mu \mathrm{m}) / f_{v}(100 \mu \mathrm{m})$. Among other effects, this may be attributed to a less efficient photoelectric heating due to charged grains (Malhotra et al. 2001; Croxall et al. 2012), or to a UV-photon screening from large amounts of dust as in ultra-luminous IR galaxies (ULIRGs) (Abel et al. 2009; Graciá-Carpio et al. 2011). With observed FIR-color 1, Haro 11 falls within the large spread of $L_{[\mathrm{C} \mathrm{II}]} / L_{\mathrm{TIR}}$ values and is not particularly [C II] deficient nor [C II] excessive, as often seen in low-metallicity galaxies (Madden et al., in prep.).

\subsection{Photometry data}

For the discussion on the SED of Haro 11 in Sect. 7.1, we use broad-band data available from the X-ray to the radio regime, to assess how the models perform in reproducing the continuum emission. The photometry is taken from NED. In addition, we use the Spitzer/MIPS photometry from Bendo et al. (2012), the Herschel/PACS and SPIRE photometry from

\footnotetext{
3 Note that they use $L_{\mathrm{FIR}}$, determined from IRAS fluxes with the Helou et al. (1988) formula, and that $L_{\mathrm{TIR}}$ estimated as $2 \times L_{\mathrm{FIR}}$ (Hunter et al. 2001) can be different from the true $L_{\mathrm{TIR}}$ when including submm observations.
}

Table 3. Photometry data.

\begin{tabular}{lcccc}
\hline \hline Instrument & Wavelength & $\begin{array}{c}\text { Flux } \\
(\mathrm{Jy})\end{array}$ & $\begin{array}{c}\text { Uncertainty } \\
\text { (Jy) }\end{array}$ & Reference \\
\hline Chandra & $2-10 \mathrm{keV}$ & $9.17 \mathrm{e}-09$ & $1.03 \mathrm{e}-09$ & $(1)$ \\
Chandra & $0.5-2 \mathrm{keV}$ & $3.21 \mathrm{e}-08$ & $0.43 \mathrm{e}-08$ & $(1)$ \\
ROSAT & $0.1-2 \mathrm{keV}$ & $4.41 \mathrm{e}-08$ & $0.55 \mathrm{e}-08$ & $(2)$ \\
FUSE & $1150 \AA$ & $2.30 \mathrm{e}-03$ & $0.33 \mathrm{e}-03$ & $(3)$ \\
GALEX & $1530 \AA$ & $3.63 \mathrm{e}-03$ & $7 \mathrm{e}-05$ & $(4)$ \\
GALEX & $2315 \AA$ & $4.37 \mathrm{e}-03$ & $4 \mathrm{e}-05$ & $(4)$ \\
Cousins & $0.439 \mu \mathrm{m}$ & $8.03 \mathrm{e}-03$ & $0.69 \mathrm{e}-03$ & $(5)$ \\
Cousins & $0.639 \mu \mathrm{m}$ & 0.012 & 0.001 & $(5)$ \\
2MASS & $1.25 \mu \mathrm{m}$ & 0.013 & 0.001 & $(6)$ \\
2MASS & $1.64 \mu \mathrm{m}$ & 0.013 & 0.001 & $(6)$ \\
2MASS & $2.17 \mu \mathrm{m}$ & 0.014 & 0.001 & $(6)$ \\
IRAC & $3.6 \mu \mathrm{m}$ & 0.023 & 0.002 & $(7)$ \\
IRAC & $4.5 \mu \mathrm{m}$ & 0.029 & 0.003 & $(7)$ \\
IRAC & $5.8 \mu \mathrm{m}$ & 0.073 & 0.007 & $(7)$ \\
IRAC & $8 \mu \mathrm{m}$ & 0.177 & 0.018 & $(7)$ \\
IRAS & $12 \mu \mathrm{m}$ & 0.52 & 0.02 & $(8)$ \\
MIPS & $24 \mu \mathrm{m}$ & 2.51 & 0.03 & $(9)$ \\
IRAS & $25 \mu \mathrm{m}$ & 2.49 & 0.03 & $(8)$ \\
IRAS & $60 \mu \mathrm{m}$ & 6.88 & 0.04 & $(8)$ \\
MIPS & $70 \mu \mathrm{m}$ & 4.91 & 0.49 & $(9)$ \\
PACS & $70 \mu \mathrm{m}$ & 6.08 & 0.18 & $(10)$ \\
IRAS & $100 \mu \mathrm{m}$ & 5.04 & 0.03 & $(8)$ \\
PACS & $100 \mu \mathrm{m}$ & 4.97 & 0.15 & $(10)$ \\
MIPS & $160 \mu \mathrm{m}$ & 2.01 & 0.24 & $(9)$ \\
PACS & $160 \mu \mathrm{m}$ & 2.43 & 0.12 & $(10)$ \\
SPIRE & $250 \mu \mathrm{m}$ & 0.633 & 0.042 & $(10)$ \\
SPIRE & $350 \mu \mathrm{m}$ & 0.231 & 0.013 & $(10)$ \\
SPIRE & $500 \mu \mathrm{m}$ & 0.092 & 0.008 & $(10)$ \\
LABOCA & $870 \mu \mathrm{m}$ & 0.040 & 0.006 & $(7)$ \\
\hline
\end{tabular}

Notes. (1) Hayes et al. (2007); (2) Tajer et al. (2005); (3) Grimes et al. (2009); (4) Iglesias-Páramo et al. (2006); (5) Lauberts \& Valentijn (1989); (6) Jarrett et al. (2000); (7) Galametz et al. (2009); (8) Sanders et al. (2003b); (9) Bendo et al. (2012); (10) Rémy et al. (in prep.).

Rémy et al. (in prep.), and the Spitzer/IRAC and LABOCA $870 \mu \mathrm{m}$ photometry from Galametz et al. (2009). All fluxes are summarized in Table 3.

\section{Modeling strategy}

\subsection{Identification of the model components}

We aim to model the IR emission from all 17 detected lines (except the MIR $\mathrm{H}_{2}$ lines which will be modeled in a coming paper) listed in Table 2 to understand the conditions of the various media and the dominant processes at work in this low-metallicity starburst. The ISM is very heterogeneous, and over the scale of a galaxy, various phases with a wide range of conditions are mixed together.

In our spectral dataset, we notice that Haro 11 is typified by very intense [Ne III], [Ne II], [S III], [S IV], [O III], and [N III] lines. These high-excitation lines require a hard radiation field which is naturally encountered in a compact H II region. Stars in compact H II regions are young, hence their UV radiation is harder, and SSCs are likely to contain massive stars which contribute to the production of hard photons. Moreover, the high critical densities of these lines (except the [O III] line) favor their origin at the same location. Therefore, the intensity of these lines demonstrates the importance of the dense $\mathrm{H}$ II region in the global spectrum of Haro 11. Moreover, the Herschel observations show bright $[\mathrm{O} \mathrm{I}]$ and $[\mathrm{C}$ II] lines, which are usual tracers of the neutral gas. In particular, the intensity of the $[\mathrm{O} \mathrm{I}]$ lines 
indicates the presence of dense PDRs. These two dense (ionised and neutral) phases will be investigated first since they account for the emission of most of the spectral lines. Derived physical conditions are presented in Sect. 4.

With low critical density $\left(\sim 300 \mathrm{~cm}^{-3}\right)$ and low ionisation potential $(14.5 \mathrm{eV})$, the [N II] $122 \mu \mathrm{m}$ line is a tracer of lowexcitation diffuse gas rather than the dense $\mathrm{H}$ II region. The intensity of [N II] demonstrates the need for an additional diffuse low-ionisation component that is investigated in Sect. 5. Our subsequent modeling also shows that this diffuse phase is the main contributor to the [Ne II] and [C II] lines.

Therefore, the three main phases that we identify as necessary model components to account for the observed emission lines are: 1) a dense ionised phase (Sect. 4.1), 2) PDR (Sect. 4.2), 3) a diffuse low-ionisation medium (Sect. 5).

In addition, we discuss the possible presence of other model components that may influence the line predictions. The detection of the high-excitation [O IV] line is the driver of a discussion on the role of X-rays and XDRs in Sect. 6.3. The [O III] line, which is the brighest of all MIR-FIR lines, has high excitation potential $(35.1 \mathrm{eV})$ and low critical density $\left(\sim 500 \mathrm{~cm}^{-3}\right)$. Although it is accounted for by the dense H II model, it may originate as well from diffuse high-excitation medium that we do not model but briefly discuss in Sect. 6.2. Finally, we investigate the influence of magnetic fields on the cloud density profile and their impact on the PDR predictions in Sect. 6.1.

\subsection{Method}

We use the 1D spectral synthesis code Cloudy v10.00, last described by Ferland et al. (1998), which includes photoionisation and photodissociation treatment (Abel et al. 2005), to model the multiple phases from which the emission lines originate. Since Haro 11 is not resolved in most of the Spitzer and Herschel bands, we have no spatial constraints. Thus, the model is reduced to a central source of energy surrounded by a spherical cloud. In the case of Haro 11, this central source is a starburst. The dense ionised gas that is closest to the central source with respect to the other phases that we model separately will yield the best constraints on the properties of the stellar cluster that is powering the model. Therefore we first derive the best fit model concentrating on the dense $\mathrm{H}$ II region diagnostics, which are the [Ne II] $12.8 \mu \mathrm{m}$, [Ne III] $15.6 \mu \mathrm{m}$, [S IV] $10.5 \mu \mathrm{m}$, [S III] $18.7 \mu \mathrm{m}$ and $33.5 \mu \mathrm{m}$, and [N III] $57 \mu \mathrm{m}$ lines. Moreover, the interpretation of these ionic lines is less ambiguous than, for example, the [C II] line. Following this step, we will assess what this model predicts for the PDR phase.

The starburst ionises the inner edge of the cloud, where the $\mathrm{H}$ II region begins, and the radiative transfer is computed step by step progressively into the cloud. The distance from the source to the inner edge of the cloud is the inner radius $\left(r_{\text {in }}\right)$. Depending on where the calculation is stopped, the ionised, atomic, and molecular phases can be treated as the model is computed within one Cloudy model. Here we stop the models until the molecular fraction $\mathrm{H}_{2} / \mathrm{H}$ reaches $50 \%$ such that both the ionised and PDR phases are computed within the same model. We opt to impose pressure equilibrium throughout the models. This is particularly important for the density profile between different phases. We will come back to this assumption in Sect. 4.2.

\subsubsection{Parameter grid of the dense $\mathrm{H} \|$ region}

We vary the following 3 physical parameters in order to calculate our model grid: the age of the burst $\left(A_{\text {burst }}\right)$, the initial hydrogen density at the inner edge of the cloud $\left(n_{\mathrm{H}}\right)$, and $r_{\text {in }}$, where the

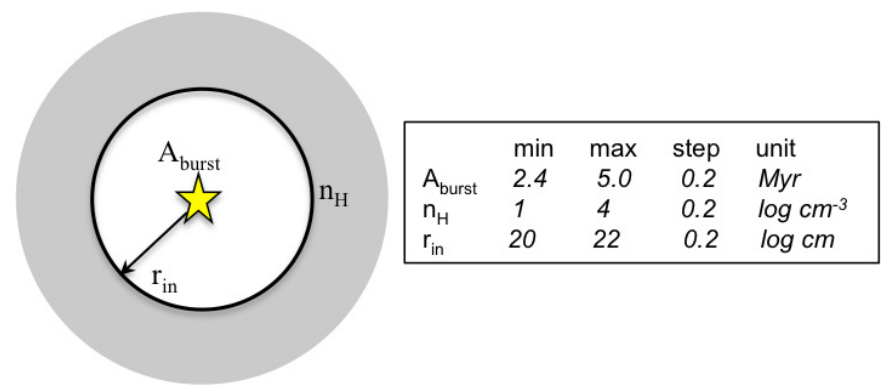

Fig. 5. Geometry of the Cloudy model with the three free parameter that we vary in the grid: the age of the burst $\left(A_{\text {burst }}\right)$, inner radius $\left(r_{\text {in }}\right)$, and initial hydrogen density $\left(n_{\mathrm{H}}\right)$. Parameter boundaries and step sizes are also indicated.

calculation starts (see Fig. 5 for an illustration). The age is varied from 2.4 to $5.0 \mathrm{Myr}$ (Sect. 4.1.2), $r_{\text {in }}$ from $10^{20.0}$ to $10^{22.0} \mathrm{~cm}$ (or $30 \mathrm{pc}$ to $3 \mathrm{kpc}$, Sect. 4.1.3), and $n_{\mathrm{H}}$ from $10^{1}$ to $10^{4} \mathrm{~cm}^{-3}$ (Sect. 4.1.1). The step size for $A_{\text {burst }}$ is $0.2 \mathrm{Myr}$ (linear step), and for $r_{\text {in }}$ and $n_{\mathrm{H}}$, we use 0.2 step sizes on a logarithmic scale.

\subsection{2. $\chi^{2}$ evaluation}

In order to find a solution for this ionised phase, we aim first to reproduce as best as possible the intensities of the ionic lines. For each individual model, the goodness of the fit is evaluated by computing the reduced $\chi^{2}$. The $\chi^{2}$ is measured from a set of selected lines. The lines we consider to constrain the fit are the following: [Ne II] $12.8 \mu \mathrm{m}$, [Ne III] $15.6 \mu \mathrm{m}$, [S IV] $10.5 \mu \mathrm{m}$, [S III] $18.7 \mu \mathrm{m}$, and $33.5 \mu \mathrm{m}$, because they are the brightest and relatively well understood, as well as the [N III] $57 \mu \mathrm{m}$ line from PACS which is also bright and expected to arise from the same medium. With 6 observations and 3 free parameters to fit, the degree of freedom is $N=n_{\mathrm{obs}}-n_{\text {param }}=3$. Best fit models are determined by minimising the reduced $\chi^{2}$. There are several lines that originate in part from the dense ionised gas that we do not consider when calculating the $\chi^{2}$, although we do discuss how the model predictions of these lines compare to the observations. We do not consider the high-excitation [O IV] line because it is faint and can be excited by several mechanisms (see Sect. 6.3). We do not include the [O III] and [N II] lines here as constraints for the dense $\mathrm{H}$ II region because our subsequent modeling shows that a fraction of these lines arises from a low density ionised medium which we consider in a later step (see Sect. 5).

\subsection{Setting the input fixed parameters}

\subsubsection{Incident radiation field}

We use Starburst99 v6.0.2 (Leitherer et al. 2010) to reproduce the incident, wavelength-dependent radiation field density of Haro 11. Following the UV-optical studies by Hayes et al. (2007) and Grimes et al. (2007), we assume a Salpeter IMF from 0.1 to $100 M_{\odot}$ and stellar tracks from Padova AGB with metallicity $Z=0.004$. According to Adamo et al. (2010), the stellar population of Haro 11 is very young. The present starburst started less than $40 \mathrm{Myr}$ ago, and shows a formation peak at about 3.5 Myr. Knot B is dominated by a young starburst of 3.5 Myr, and knot C contains an older starburst of 9.5 Myr (Fig. 1). Therefore, we select the stellar spectrum of an instantaneous burst of a few Myr, letting the age of the burst be a free parameter, varying from 2.4 to 5.0 Myr. We also add to this the 
spectrum of an instantaneous burst of 9.5 Myr to account for the slightly older stellar population. We keep this age fixed since the ionising part of the stellar spectrum does not vary significantly for ages larger than $5 \mathrm{Myr}$, and will therefore not significantly affect the model predictions of the ionised gas. This starburst spectrum dictates the shape of the input energy source in Cloudy. The incident radiation field on the cloud is shown in purple in Fig. 12. For the input luminosity of the central source, we take the total observed infrared luminosity of Haro 11: $L_{\mathrm{TIR}}=1.4 \times 10^{11} L_{\odot}$. This approximation assumes an energy balance between the UV and IR domains, i.e. that all of the UV photons are reprocessed in the IR by the gas and the dust. The emergent SED (bottom panel of Fig. 12) shows that this approximation is reasonable since only $20 \%$ of the total emergent luminosity is emitted in the UV-optical, and $80 \%$ in the IR. We discuss in more details the energy balance in Sect. 7.1.

Haro 11 is detected in X-rays (e.g. Grimes et al. 2007). Previous studies on X-ray observations indicate the presence of $\operatorname{soft}\left(L_{0.5-2 \mathrm{kev}}=1.9 \times 10^{8} L_{\odot}\right)$ and hard $\left(L_{2-10 \mathrm{kev}}=2.6 \times 10^{8} L_{\odot}\right)$ $\mathrm{X}$-rays. Therefore we add to the input SED an X-ray component that reproduces the shape of the observed X-ray spectrum and of total luminosity $5 \times 10^{8} L_{\odot}$. The effects of the X-rays in the models are discussed in Sect. 6.3.

\subsubsection{Elemental abundances}

The elemental abundances directly impact on the predicted line intensities. To match the observed abundances in Haro 11 as closely as possible, we use the abundances in the literature. Abundances of oxygen, nitrogen, neon, sulphur, and argon were obtained in knots B and C of Haro 11 by Guseva et al. (2012) using VLT/X-shooter observations in the optical. In particular, they find an oxygen abundance $12+\log (\mathrm{O} / \mathrm{H})$ of 8.1 in knot $\mathrm{B}$ and 8.3 in knot $\mathrm{C}$, giving a $\mathrm{O} / \mathrm{H}$ twice as large as the value $12+\log (\mathrm{O} / \mathrm{H})=7.9$ previously found by Bergvall \& Östlin (2002), altering the determination of the metallicity from $\sim 1 / 6$ to $1 / 3 Z_{\odot}$. We use the abundances in Guseva et al. (2012), averaged over the two knots, which have similar luminosities, for our calculations. Uncertainties on these abundances are $10 \%$. For neon and sulphur, Guseva et al. (2012) find similar values to those found in Wu et al. (2008), derived from Spitzer data. The relatively high neon abundance is typical of BCDs (Izotov \& Thuan 1999). For nitrogen, such high abundance is observed in a few BCDs (e.g. Thuan et al. 1996; Izotov \& Thuan 1998; Pustilnik et al. 2004), and can be explained by the presence of Wolf-Rayet stars (Bergvall \& Östlin 2002), which inject matter enriched in $\mathrm{N}$ and $\mathrm{C}$ into the ISM. For the other elements, we use gas-phase relative abundances typical of $\mathrm{H}$ II regions, based on an average of the abundances in the Orion Nebula determined by Baldwin et al. (1991), Rubin et al. (1991), Osterbrock et al. (1992), Rubin et al. (1993), and scaling them to the metallicity of Haro 11. The resulting carbon abundance is $\log (\mathrm{C} / \mathrm{O})=-0.15$, which is relatively high compared to Izotov \& Thuan (1999), where $\log (\mathrm{C} / \mathrm{O}) \sim-0.5$. However, Bergvall et al. (2006) find that the ratio of $\mathrm{C}$ and $\mathrm{O}$ column densities is $\sim-0.3(\mathrm{log})$ from the analysis of absorption lines in IUE and FUSE data. Such high value may indicate an enrichment in $\mathrm{C}$ and agrees with our adopted carbon abundance (assuming Orion-like $\mathrm{C} / \mathrm{O}$ ) within the uncertainties. For silicon, its abundance $\mathrm{Si} / \mathrm{O}$ is low in the Orion abundance set compared to that usually found in BCDs, therefore we use the average abundance of $\log (\mathrm{Si} / \mathrm{O})=-1.5$ from Izotov \& Thuan (1999). We consider the uncertainty on these theoretical abundances to be of a factor of 2 . Table 4 summarizes the elemental abundances (and uncertainties) we use in
Table 4. Elemental abundances set in Cloudy.

\begin{tabular}{ll}
\hline \hline Gas-phase composition & Abundance (uncertainty) in log \\
\hline $\mathrm{O} / \mathrm{H}$ & $-3.79(0.14)$ \\
$\mathrm{N} / \mathrm{H}$ & $-4.64(0.12)$ \\
$\mathrm{Ne} / \mathrm{H}$ & $-4.44(0.07)$ \\
$\mathrm{S} / \mathrm{H}$ & $-5.57(0.10)$ \\
$\mathrm{Ar} / \mathrm{H}$ & $-6.20(0.13)$ \\
$\mathrm{C} / \mathrm{H}$ & $-3.92(0.3)$ \\
$\mathrm{Si} / \mathrm{H}$ & $-5.80(0.3)$ \\
$\mathrm{Fe} / \mathrm{H}$ & $-5.92(0.3)$ \\
\hline Grain composition & Mass fraction \\
\hline Carbonaceous & $36 \%$ \\
Silicate & $60 \%$ \\
PAH & $4 \%$ \\
\hline Dust-to-gas mass ratio & $2.64 \times 10^{-3}$ \\
\hline
\end{tabular}

Notes. Abundances of oxygen, nitrogen, neon, sulphur, and argon are from Guseva et al. (2012), silicon from Izotov \& Thuan (1999), and the others are average abundances of the Orion Nebula determined by Baldwin et al. (1991), Rubin et al. (1991), Osterbrock et al. (1992), Rubin et al. (1993), scaled to the metallicity of Haro 11. The main elemental composition $\mathrm{O}: \mathrm{C}: \mathrm{Si}: \mathrm{Fe}$ in the grains is 4:9:1:1.

Cloudy. The lack of measured abundances for the other elements limits their use to constrain the models (e.g. Fe, Si).

\subsubsection{Dust properties}

It is important to accurately set the dust properties for two reasons: (1) dust plays an important role in heating the gas through the photoelectric effect, which depends on the dust abundance and grain size distribution, and (2) the radiative transfer is strongly influenced by the properties of the grains. We first tried using the standard MRN grain size distribution (Mathis et al. 1977), which failed to reproduce the observed dust emission in the MIR, falling below the photometry data by more than an order of magnitude. The MRN distribution underpredicts the number of small grains (minimum grain radius of $5 \mathrm{~nm}$ ) and is usually not appropriate for modeling the MIR emission in galaxies. Standard dust models such as Draine \& Li (2001) and Zubko et al. (2004) do include more small grains than the standard MRN model. Dust modeling of dwarf galaxies (e.g. Galliano et al. 2003, 2005; Galametz et al. 2011) also demonstrates the need for an increased abundance of smaller grains. Therefore, in Cloudy, we use the MRN distribution extended down to $1.1 \mathrm{~nm}$ to account for the very small grains. Other grain size distributions have been used to model the IR emission of galaxies, such as grain shattering distributions (Dopita et al. 2005), that we discuss in Sect. 7.1.2. We also use PAHs as described in Abel et al. (2008). Both grains and PAH abundances are scaled to the metallicity of Haro $11\left(1 / 3 Z_{\odot}\right)$, fixing the dust-to-gas mass ratio $(\mathrm{D} / \mathrm{G})$ to $2.64 \times 10^{-3}(\sim 1 / 400$, the Galactic $\mathrm{D} / \mathrm{G}$ is $\sim 1 / 150)$. This yields a ratio of visual extinction to hydrogen column density, $A_{\mathrm{V}} / N(\mathrm{H})$, of $10^{-22} \mathrm{mag} \mathrm{cm}^{-2}$.

\subsection{Limitations of the approach}

Our first limitation lies on the geometry of our modeling that we aim to constrain as much as possible with diagnostics from a large set of spectral lines. Some of the derived parameters of the compact H II region are strongly dependent on the (simple) geometry we impose on the model, which we will see is a recurring theme throughout the paper. The parameters that 
are best constrained, irrespective of the geometry, are the electron density, temperature and mass of ionised gas. As found in Sect. 4.1.3, the geometrically thin ionised gas shell, in combination with the large inner radius $(\sim 1 \mathrm{kpc})$ that describes the model solution of the dense H II region, seems physically implausible. Despite the geometry of the model, we refer to it with the term "compact" since this model component represents what is observationally described as a compact H II region. This is a direct result of assuming a single region with a single central source. Although we are aware that the knots B and C of Haro 11 are spatially separated in the optical, we do not have the spatial resolution in the Spitzer and Herschel observations to treat them separately. If, for example, we were to construct a model as the sum of several regions - as seems reasonable when perusing the optical images - the inner radius of each region would have to decrease in order to obtain a similar effective $U$ parameter, while the shell thickness would increase in order to obtain the same gas mass, thus evolving towards a model with a more geometrically thick layout. It is impossible to obtain reliable and reproducible results using such a complex model, without better observational constraints on the actual source distribution, i.e. spatially resolved spectroscopy. In this paper we focus on the derived parameters that are least sensitive on the actual assumed geometry. In Sect. 7.1, we discuss the spectral energy distribution resulting from the combination of models of the various phases in terms of energy balance, but we do not pretend to accurately fit the total SED because of these geometry issues.

Our second limitation lies in the evalutation of the goodness of the fits. It is mainly weighted by the elemental abundance uncertainties, which can be up to a factor of 2 , and by the observational uncertainties (up to $40 \%$ ). Therefore, when comparing the model predictions to observed intensities, we aim for a match within a factor of 2 .

\section{A model of the $\mathrm{H}$ II region and PDR: $\mathscr{C}$}

\subsection{The compact $H$ II region}

The results of the computed grid are displayed in Fig. 6. It shows the intensity predictions of the compact model labeled $\mathscr{C}$ for each ionic line as a function of density, $n_{\mathrm{H}}$, and inner radius, $r_{\text {in }}$, for a starburst age of 3.7 Myr (age of the best fit model, see Sect. 4.1.2). In this section, we discuss the influence of each grid parameter on the model predictions and possible solution values. These parameters are linked via the quantity $U$, which is the ionisation parameter, defined as the dimensionless ratio of the incident ionising photon surface density to the hydrogen density $n_{\mathrm{H}}$ : $U=\frac{Q(\mathrm{H})}{4 \pi r_{\mathrm{in}}^{2} n_{\mathrm{H}} c}$, where $Q(\mathrm{H})$ is the rate of hydrogen-ionising photons striking the illuminated face and $c$ is the velocity of light. $U$ describes how many ionising photons arrive per atom.

\subsubsection{The density of the gas}

In general terms the predicted line intensities are dependent on the gas density through both the critical density of each atom, and the ionisation parameter, $U$. Line intensities increase with increasing gas density until the critical density of a fine-structure line is reached. Above such critical density, the de-excitation process is dominated by collisions rather than radiative decay. Therefore the maximum gas density is well determined by the critical density of the brightest line tracers. The gas density also plays an important role in determining the population of the different ionisation stages. The ionisation parameter, $U$, is inversely proportional to the density (bottom right panel of Fig. 6).

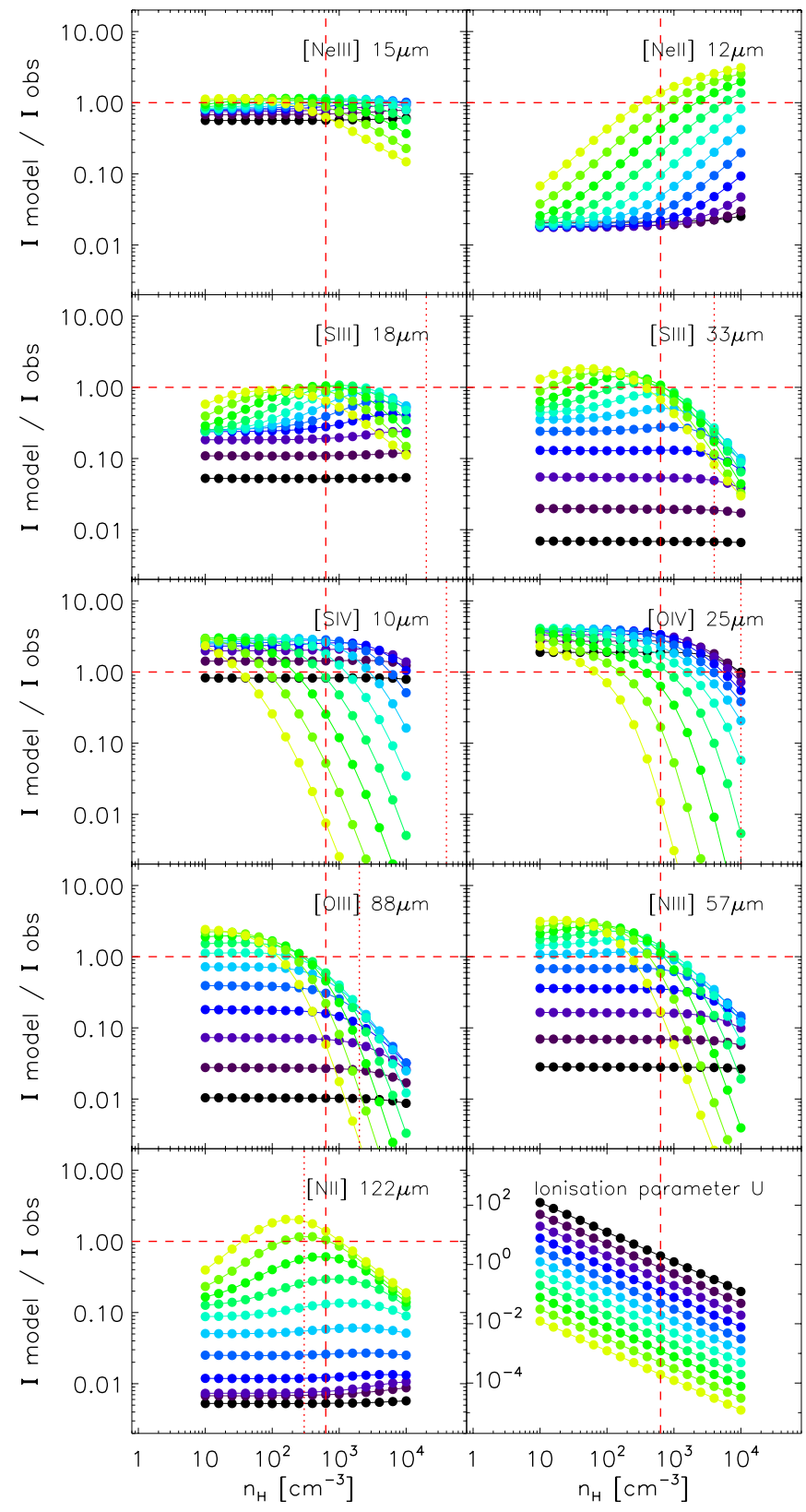

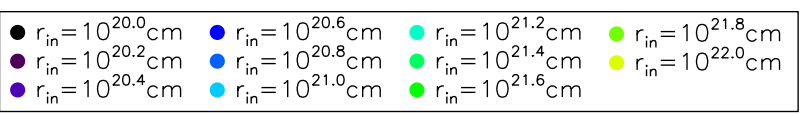

Fig. 6. Predicted intensities for model $\mathscr{C}\left(I_{\text {model }}\right)$ normalised by their observed value $\left(I_{\mathrm{obs}}\right)$ for all ionic lines considered, as a function of $n_{\mathrm{H}}$ and $r_{\text {in }}$ for a given starburst age (of 3.7 Myr). The vertical dashed line shows the best fit model density $n_{\mathrm{H}}=10^{2.8} \mathrm{~cm}^{-3}$, and the vertical dotted line the critical density of the ionic specie, if within the range displayed. The best fit inner radius is $r_{\mathrm{in}}=10^{21.4} \mathrm{~cm}$ (green symbols). (This figure is available in color in electronic form.)

We find that the minimum $\chi^{2}$ occurs for a solution with a density around $10^{2.8} \mathrm{~cm}^{-3}$ (dashed vertical line in Fig. 6). This derived gas density compares well with density diagnostics proposed in the literature, for example, based on the ratio of the [S III] lines at 18.7 and $33.5 \mu \mathrm{m}$ (Houck et al. 1984). This ratio is sensitive to the density since these lines originate from the same ion but with significantly different critical densities (Table 2). From Abel et al. (2005) and requiring $\frac{[\mathrm{S} \text { III] } 18 \mu \mathrm{m}}{[\mathrm{S} \text { III] } 33 \mu \mathrm{m}} \sim 1.0$, we obtain a density of the ionised gas of $n_{\mathrm{H}}=10^{2.7} \mathrm{~cm}^{-3}$. The value 
of the electron density predicted by the diagnostic plot in Houck et al. (1984) is also around $10^{2.8} \mathrm{~cm}^{-3}$. Following the method from Kingsburgh \& Barlow $(1992,1994)$ who use the programs EQUIB and RATIO (developed at UCL by Howarth and modified by Adams), we also find $n_{\mathrm{H}} \sim 10^{2.8} \mathrm{~cm}^{-3}$.

\subsubsection{The age of the burst}

The spectrum of the starburst (intensity and shape) changes drastically with time in the age range considered here. The age of the burst sets the hardness of the incident radiation field. Figure 7 shows line ratios of [Ne III]/[Ne II], [O IV]/[Ne III], [O III]/[N III], $[\mathrm{O} \mathrm{III}] /[\mathrm{S} \mathrm{IV}]$, and [S IV]/[S III] $18.7 \mu \mathrm{m}$ as a function of burst age. The observed ratios agree with an age of burst of 3.5-5 Myr. These values are also in agreement with the age of $3.5 \mathrm{Myr}$ for the brightest knot $\mathrm{B}$ and $9 \mathrm{Myr}$ for knot $\mathrm{C}$, and a mixture of very young and older bursts, found in Adamo et al. (2010). After $5 \mathrm{Myr}$, the ionising part of the burst spectrum decreases, the high ionisation lines start to disappear, and in particular the [Ne III]/[Ne II] ratio drops off (bottom panel of Fig. 7). This is why we do not compute models with $A_{\text {burst }}>5$ Myr in our grid. The best fit on the neon lines favours a relatively young burst, of about 3 Myr. However, the emission of the highest excitation potential [O IV] line $(54.9 \mathrm{eV})$ is rather favoured by models with ages between 3.7 and 4.7 Myr. Indeed this time frame corresponds to the time needed for the onset of WolfRayet stars. A burst age of 4.7 Myr is also a good match to the $[\mathrm{Ne}$ III] $][\mathrm{Ne}$ II] ratio, although it does not agree with the intensity of the [S IV] line, which is one of the lines that has the highest weight in the $\chi^{2}$. The best single solution model in that case has an age of burst of 3.7 Myr.

\subsubsection{The inner radius}

All lines are sensitive to the inner radius $r_{\text {in }}$. Its choice has no direct influence on the hardness of the radiation field, but rather on its intensity. With increasing radius, the total input luminosity is distributed over a larger surface, decreasing the number of ionising photons per surface area, and therefore the ionisation rate. $U$ decreases as the inverse square of the radius. Intensities of species with a high ionisation potential, such as [O IV], decrease with increasing $r_{\text {in }}$, and the inverse is observed for low ionisation species such as [N II]. For $r_{\text {in }}<10^{21.0} \mathrm{~cm}$, atoms are preferably present in high ionisation states. Models with such small $r_{\text {in }}$ often predict too much [O IV] and [S IV], and not enough [Ne II] and [S III]. [Ne III] and [S IV] intensities are constant at low $r_{\text {in }}$ and then drop with increasing $r_{\text {in }}$. [S III], [N III] and [O III] intensities increase with low $r_{\text {in }}\left(<10^{21.5} \mathrm{~cm}\right)$ because of an ionisation balance between $\mathrm{S}^{2+}, \mathrm{N}^{2+}, \mathrm{O}^{2+}$ and $\mathrm{S}^{3+}, \mathrm{N}^{3+}, \mathrm{O}^{3+}$. Then their intensities drop with increasing $r_{\text {in }}$. The opposite effect is observed for the lower ionisation state species such as [Ne II] and [N II] which increase with $r_{\text {in }}$.

The observations are best matched for an inner radius of $10^{21.4} \mathrm{~cm}(800 \mathrm{pc})$. At that distance, the model cloud is a very thin shell of large radius, at the center of which resides the central starburst. The resulting geometry is plane-parallel. This is not a realistic geometry, as discussed in Sect. 3.4, but we do not aim to model the spatial structures in Haro 11.

\subsubsection{Parameters of the best fit model $\mathscr{C}_{\text {HII }}$}

We have minimised the $\chi^{2}$ to find the parameters of the best fit model for the compact $\mathrm{H}$ II region described above. Figure 8

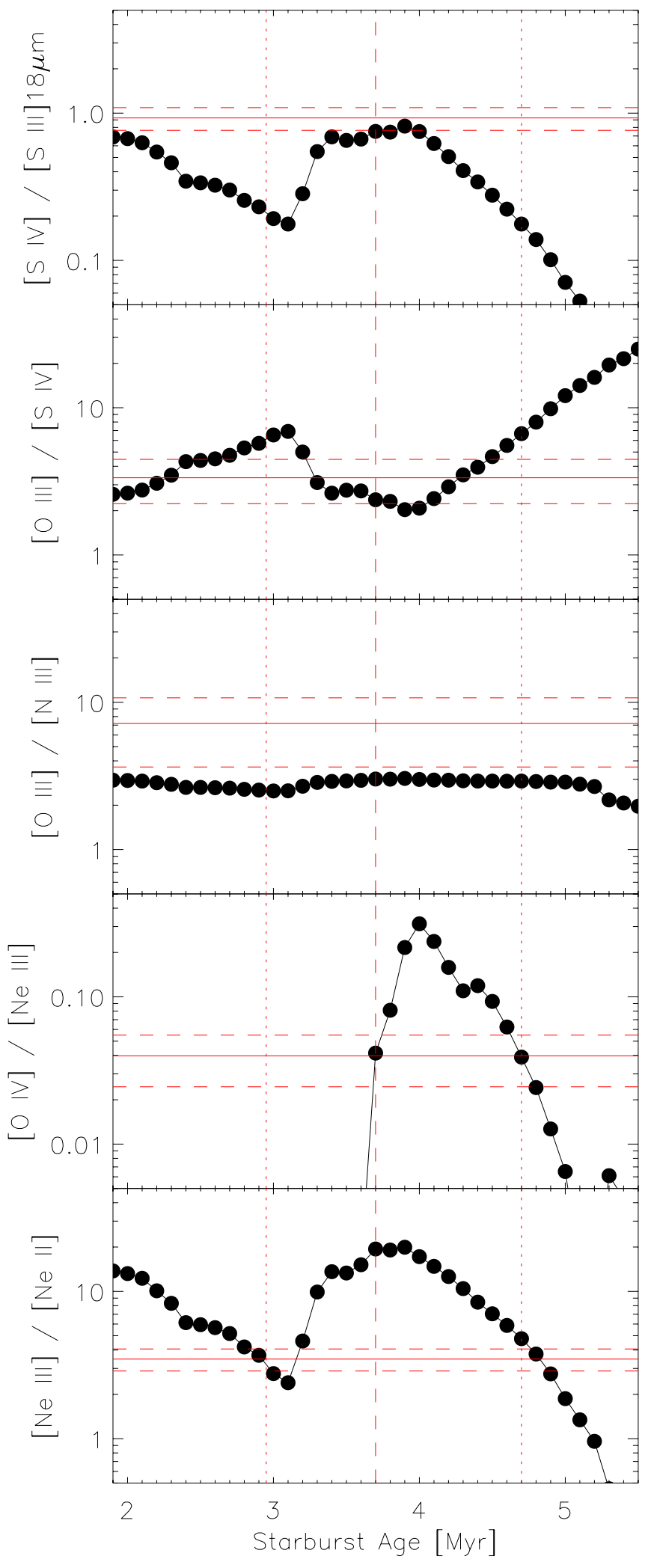

Fig. 7. Model predicted intensity ratios as a function of burst age $A_{\text {burst }}$, for a hydrogen density of $10^{2.8} \mathrm{~cm}^{-3}$, and inner radius of $10^{21.4} \mathrm{~cm}$. The true burst age of these models is an average of $A_{\text {burst }}$ and the older stellar population of $9.5 \mathrm{Myr}$, although the latter has a negligible influence on the high-ionisation lines considered here. The horizontal solid lines represent the observed ratios and the dashed lines the uncertainties on the ratios. The vertical dashed line shows the solution burst age of 3.7 Myr, and the vertical dotted lines the age of $3 \mathrm{Myr}$ and $4.7 \mathrm{Myr}$ that would better match the $[\mathrm{Ne} \mathrm{III}] /[\mathrm{Ne} \mathrm{II}]$ ratio. (This figure is available in color in electronic form.) 

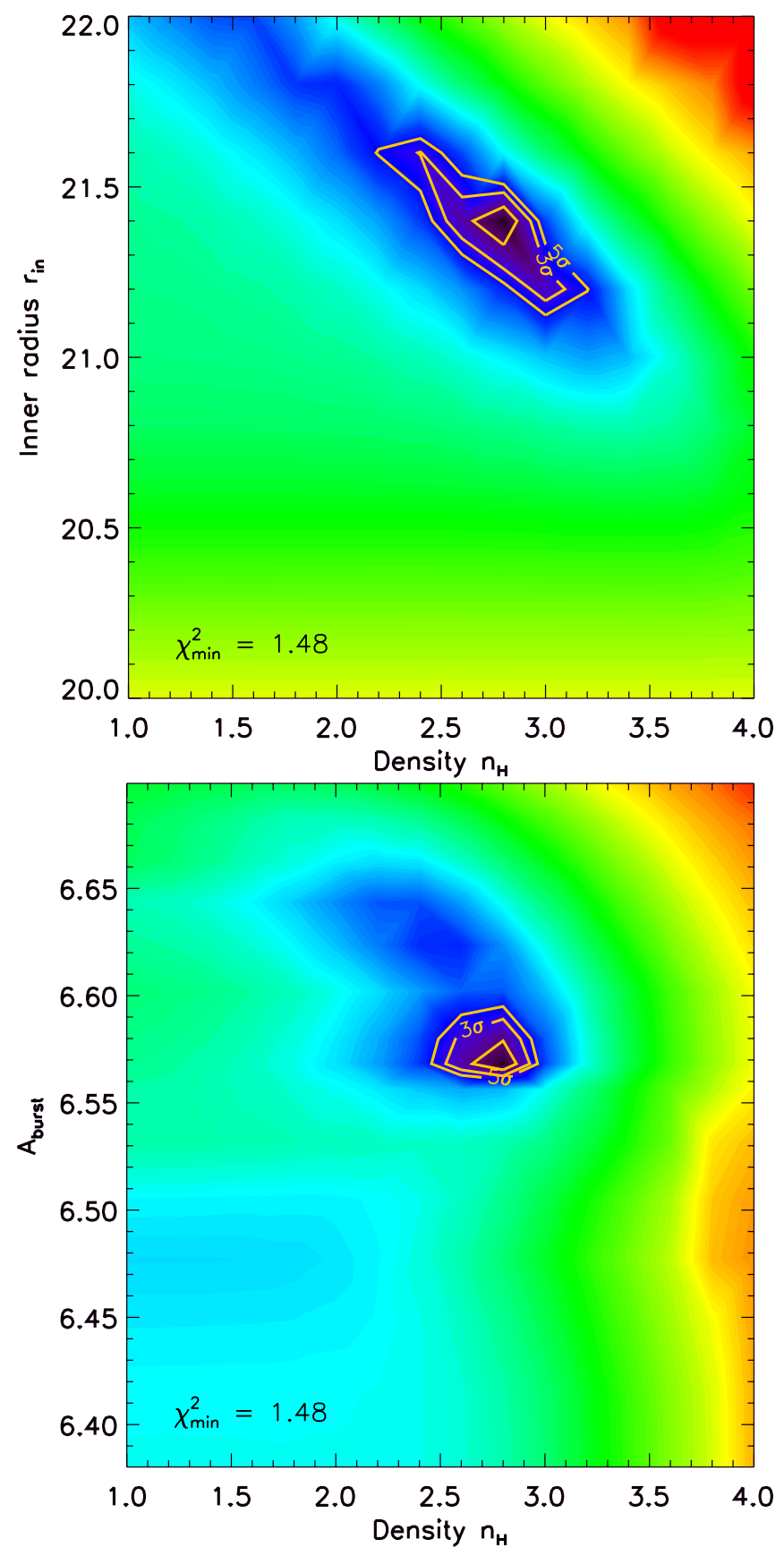

Fig. 8. Contour plots of the $\chi^{2}$ values of the $3 \mathrm{D}$ grid (log scale). The parameters are given in logarithmic values. Units are $\mathrm{cm}^{-3}$ for the density, $\mathrm{yr}$ for the burst age, and $\mathrm{cm}$ for the inner radius. The minimum reduced $\chi^{2}$ found for $\mathscr{C}_{\mathrm{HII}}$ is $\chi_{\min }^{2}=1.48$. Contour levels at $1-\sigma, 3-\sigma$, and 5- $\sigma$ from the minimum $\chi^{2}$ are displayed in orange. There are 5 other models within 3- $\sigma$ of $\chi_{\min }^{2}$. The red color on the plots correspond to $>1000-\sigma$ level. Top: $\chi^{2}$ contours in the $n_{\mathrm{H}}-r_{\text {in }}$ plane, for $A_{\text {burst }}=3.7 \mathrm{Myr}$ (or 6.57 in log). Bottom: $\chi^{2}$ contours in the $n_{\mathrm{H}}-A_{\text {burst }}$ plane, for $r_{\text {in }}=10^{21.4} \mathrm{~cm}$. (This figure is available in color in electronic form.)

shows contour plots of the reduced $\chi^{2}$ computed for our grid of models. Contour levels at $1-\sigma, 3-\sigma$, and $5-\sigma$ from the minimum $\chi^{2}$ are displayed in orange. The intensities of the spectral lines included in the fit are best reproduced with the parameters: $n_{\mathrm{H}}=10^{2.8} \mathrm{~cm}^{-3}, A_{\text {burst }}=3.7 \mathrm{Myr}$, and $r_{\text {in }}=10^{21.4} \mathrm{~cm}$. The ionisation parameter is $U=10^{-2.5}$. The reduced $\chi^{2}$ of this model solution, which has the minimum $\chi^{2}$, is 1.48 . This value is close to 1 , indicating that this model describes the data well, with a probability of $\sim 70 \%$. For clarity throughout the following sections, we will refer to this model solution of the compact H II region as $\mathscr{C}_{\text {HII }}$.

With $\mathscr{C}_{\mathrm{HII}}$, the strongest MIR line, [Ne III], is reproduced within $15 \%$, as well as the [O IV] line. The two [S III] lines are achieved within $5 \%$ and their ratio within $1 \%$. The modeled [S IV] and [Ar III] lines deviate by $20 \%$. The [N III] line is reproduced within $40 \%$. The [Ne II] line, however, is under-predicted by a factor of 5 by $\mathscr{C}_{\mathrm{HII}}$.

We then compare the line intensities predicted by $\mathscr{C}_{\text {HII }}$ to the PACS lines, [O III] and [N II] $122 \mu \mathrm{m}$, which were not used to constrain the model fit since they can originate from less dense ionised gas. We find that [O III] is under-predicted by a factor of 2 , which is within our expectations, while the [N II] $122 \mu \mathrm{m}$ is under-predicted by a factor of 4 . The dense $\mathrm{H}$ II region is not the primary source of [N II]. The prediction for the [N II] $205 \mu \mathrm{m}$ line falls below its derived upper limit.

We discuss the origin of the [Ne II] and [N II] lines from diffuse low-ionisation gas in Sect. 5 and the possible origin of the [O III] emission from diffuse high-ionisation gas in Sect. 5.

We also find that $\mathscr{C}_{\mathrm{HII}}$ contributes very little to the [O I] emission (3\%), as expected since the atomic oxygen is a tracer of the neutral gas. $\mathscr{C}_{\text {HII }}$ shows very little contribution to the [C II] $(<1 \%)$ and [Si II] $(11 \%)$ lines as well. The energy required to create $\mathrm{C}^{++}$ions is $24.4 \mathrm{eV}$, close to that of $\mathrm{S}^{++}$, therefore when the radiation field is sufficiently hard, as is the case inside the $\mathrm{H}$ II region, $\mathrm{C}$ is doubly rather than singly ionised.

\subsection{The dense photodissociation regions: $\mathscr{C}_{P D R}$}

We subsequently investigate the properties of the neutral gas and compare model predictions to the [C II] and [O I] 63 and $145 \mu \mathrm{m}$ lines. To this end, we extend $\mathscr{C}_{\text {HII }}$ into the atomic phase and the outer envelope of the molecular phase, until the molecular fraction $\mathrm{H}_{2} / \mathrm{H}$ reaches $99 \%$. The modeled shell is fully covered by the gas. The ionised cloud, with average density of $10^{2.8} \mathrm{~cm}^{-3}$, transitions from the $\mathrm{HII}$ region into a dense PDR, which is where the $[\mathrm{O} I]$ originates. This transition between these two phases in a constant pressure model translates into a jump of the gas density at the ionisation front. With this model, the average density of the PDR is $10^{5.1} \mathrm{~cm}^{-3}$. The radiation field striking the PDR is: $G_{0}=3.5 \times 10^{3}$ Habing unit $\left(1.6 \times 10^{-3} \mathrm{erg} \mathrm{cm}^{-2} \mathrm{~s}^{-1}\right)$. The heating of the gas in the PDR is dominated by grain photoelectric heating $(85 \%)$, and there is a second order contribution from collisions with $\mathrm{H}^{0}, \mathrm{He}$, and $\mathrm{H}_{2}$. The cooling is done by radiative de-excitation mainly of the [O I] $63 \mu \mathrm{m}(65 \%)$, [Si II] $(20 \%)$, [C II] $157 \mu \mathrm{m}$ and [O I] $145 \mu \mathrm{m}(<7 \%$ each) lines. At such low $A_{\mathrm{v}}<1$, the [O I] lines are optically thin.

The [O I] 63 and $145 \mu \mathrm{m}$ absolute intensities are overestimated by a factor of 10 , while their ratio is reproduced within $15 \%$ by this dense PDR component. Along with the [O I] $63 \mu \mathrm{m}$ line, [C II] $157 \mu \mathrm{m}$ and [Si II] $35 \mu \mathrm{m}$ are the brightest PDR lines. While the [Si II] line intensity is over-predicted by a factor of 2 by the dense model, the [C II] line agrees with the observation within $10 \%$.

The consistent over-prediction of the two [O I] lines may be explained by the fact that, in reality, the dense PDR does not cover the full dense $\mathrm{HII}$ region. Assuming that all of the [O I] arises from a single dense phase (and not the diffuse gas, see Sect. 5.3), we reduce the covering factor of the dense PDR to only $10 \%$, which is a proxy for dense clumps (Hunter et al. 2001; Groves et al. 2008). The PDR model with a covering factor of $1 / 10$ matches the observed [O I] emission, but under-estimates the $[\mathrm{C} \mathrm{II}]$ emission by an order of magnitude. The main conclusion from this is that the dense PDR component cannot account 
for the bulk of the [C II] emission observed in Haro 11, but only for $10 \%$. This mismatch for the [C II] is due to the relatively low critical density of [C II] $\left(2 \times 10^{3} \mathrm{~cm}^{-3}\right)$ compared to the high density reached in the PDR $\left(n_{\mathrm{PDR}}=10^{5.1} \mathrm{~cm}^{-3}\right)$, generated by the pressure equilibrium and the drop of gas temperature between the ionised and neutral phases. Indeed, Cloudy PDR models with constant densities of $\sim 10^{3.5} \mathrm{~cm}^{-3}$ and a covering factor of unity would reproduce the absolute intensity of the two [O I] lines simultaneously, and yet under-predict the [C II] by a factor of 2 . Moreover, the H I mass of such models would exceed the upper limit of $10^{8} M_{\odot}$ found by Bergvall et al. (2000).

The small fraction of [C II] that originates from the dense PDR is at odds with the finding of Bergvall et al. (2000), who estimate that more than $80 \%$ of the observed [C II] in Haro 11 comes from the neutral gas. They use stellar clusters of $T_{\text {eff }} \sim$ $35000-40000 \mathrm{~K}$ to account for the [O III] $88 \mu \mathrm{m}$ emission, which contributes less than $20 \%$ to the [C II] emission, and they assume that the rest of the [C II] $(>80 \%)$ is associated with PDRs. Then, they find $n_{\mathrm{PDR}}=2 \times 10^{3} \mathrm{~cm}^{-3}$ using the Kaufman et al. (1999) model, which assumes a constant density. The much higher density that we find is compatible with the findings of Vasta et al. (2010) who analysed ISO observations with PDR models for several dwarf galaxies, including Haro 11, and find PDR densities between $10^{4}$ and $9 \times 10^{4} \mathrm{~cm}^{-3}$, and $G_{0}$ between 60 and $8 \times 10^{2}$ Habing. They also estimate that 10 to $60 \%$ of [C II] comes from the ionised gas, which is in agreement with the diffuse ionised model contribution to the [C II] line that we discuss in Sect. 5.3.

We have also explored the influence of cloud geometry on the line predictions by using the PDR model Kosma- $\tau$ (Röllig et al. 2006), in which the illumination of the cloud is external, simulating a spherical cloud structure. At corresponding values of $n_{\mathrm{H}}\left(10^{5.1} \mathrm{~cm}^{-3}\right)$ and $G_{0}\left(3.5 \times 10^{3}\right.$ Habing $)$ found with Cloudy, Kosma- $\tau$ predicts ratios of [O I] $145 \mu \mathrm{m} /[\mathrm{C} \mathrm{II}] \sim 1$ and [O I] $145 / 63 \mu \mathrm{m} \sim 0.05$. The [O I] $145 \mu \mathrm{m} /[\mathrm{C} \mathrm{II}]$ model ratios are over-predicted, as found with Cloudy, compared to our observed value of 0.08 . However, if we attribute only $10 \%$ of the [C II] emission to the PDR, then [O I] $145 \mu \mathrm{m} /[\mathrm{C} \text { II }]_{\mathrm{PDR}} \sim 0.1$ from Kosma- $\tau$, which is in line with our observations. The spherical geometry affects both [O I] and [C II] lines similarly to first order, and is not the primary reason for the discrepancy between observed and predicted [C II] intensities.

We prefer to impose the pressure equilibrium between the compact $\mathrm{H}$ II region and the dense PDR, because this gives physical insight into the presence of dense but fragmented PDRs (e.g. Hunter et al. 2001). Although the pressure equilibrium assumption is not valid on large scales (nor is the assumption of a constant density) and, in particular, is expected to break in molecular clouds, here we do not model the molecular phase. We are interested in the interface between the dense $\mathrm{H}$ II region and the PDR, the presence for which there is observational evidence. This model solution of the dense PDR with covering factor of $10 \%$ is noted $\mathscr{C}_{\text {PDR }}$. Next, we will discuss possible ways to lower the density in the PDR to account for the [C II] emission, by either combining a high density with a low density model (Sect. 5.3), representative of the multi-phase ISM of galaxies, or by including a magnetic field (Sect. 6.1).

\section{A model for the diffuse medium: $\mathscr{D}$}

\subsection{Need for a softer radiation field}

The major discrepancies between observations and model predictions in the ionised gas are seen for the [Ne II] $12.6 \mu \mathrm{m}$ and
[N II] $122 \mu \mathrm{m}$ lines, which are both under-predicted by a factor of $\sim 5$ in the dense $\mathrm{H}$ II region model $\mathscr{C}_{\mathrm{HII}}$, which is not unexpected. With excitation potentials of 14.5 and $21.6 \mathrm{eV}$ respectively, emission of $\mathrm{N}^{+}$and $\mathrm{Ne}^{+}$originates only from the ionised gas, from both the dense $\mathrm{H}$ II region and the diffuse medium. For [N II] $122 \mu \mathrm{m}, \mathscr{C}_{\text {HII }}$ does not reproduce the observed value even when varying the 3 free parameters. Only $r_{\text {in }}>10^{21.8} \mathrm{~cm}$ may agree (bottom left panel of Fig. 6). The contribution of the compact $\mathrm{H}$ II region to this line is thus marginal; mostly because the radiation field produced by the young starburst is too hard and favors the ionisation of $\mathrm{N}^{++}$rather than $\mathrm{N}^{+}$. For the [Ne II] line, a small change in the age of the burst would impact on its prediction, as discussed in Sect. 4.1.2. For the same values of density and inner radius, a model of age $3 \mathrm{Myr}$ (instead of 3.7 Myr) would agree better with the observed intensity of [Ne II]. For a burst age of 3.7 Myr, higher densities $\left(n_{\mathrm{H}}>10^{3} \mathrm{~cm}^{-3}\right)$ and inner radii $\left(r_{\text {in }}>10^{21.6} \mathrm{~cm}\right)$ are required to reproduce the observed [Ne II] (top right panel of Fig. 6). However, such values of $n_{\mathrm{H}}$ and $r_{\text {in }}$ would no longer agree with the other ionic lines. In order to reconcile the modeled [Ne II] and [N II] with their observations, a component with softer radiation field is required. This highlights the fact that one component is not enough the account for the emission of all the observed lines due to the presence of several phases in the ISM of galaxies with different properties. Our physical picture of the ISM of dwarf galaxies is of a highly permeable medium, where some of the ionising photons from the central starburst escape the $\mathrm{H}$ II region and travel large distances. This means that in dwarf galaxies, larger effective volumes can be maintained in an ionised state, compared to those of dusty starbursts.

To model this additional ionised component we have tried three different methods: (1) set the cloud further away (higher $r_{\text {in }}$ ) under the same starburst conditions, and allowing the density to be a free parameter; (2) stop $\mathscr{C}_{\mathrm{HII}}$ before it reaches the ionisation front and take the output spectrum as the input spectrum of a lower density medium; (3) opt for a softer radiation field by including a scaled local interstellar radiation field. However, all of these methods fail to reproduce the observed line intensities. Either the radiation is still too hard (case (1) and (2) when stopping too early), or too soft (case (3) and (2) when stopping too close to the ionisation front).

The radiation field that works best to explain the observed [Ne II] and [N II] lines is a representative stellar SED from the Kurucz library of relatively low temperature (see the next section). This is supported by the fact that more evolved stars producing low energy photons are present in Haro 11 (Micheva et al. 2010) but not accounted for in the compact model $\mathscr{C}$ $\left(\mathscr{C}_{\text {HII }}+\mathscr{C}_{\text {PDR }}\right)$. This older population affects mainly the emission of [N II], [Ne II], and to a lesser extent [S III].

\subsection{Properties of the model $\mathscr{D}$}

We ran constant density models of diffuse ionised gas, stopped at the ionisation front, with stellar temperature between 20000 and $40000 \mathrm{~K}$. The best fit to the [N II] $122 \mu \mathrm{m}$ and [Ne II] emission is obtained for a stellar temperature of $35000 \mathrm{~K}$. Figure 9 shows how the $[\mathrm{N} \mathrm{II}],[\mathrm{Ne}$ II], [S III], and [C II] lines vary with density for several values of $r_{\text {in }}$. We refer the reader to Sects. 4.1.1 and 4.1.3 for an interpretation of the behavior of the lines. The range of possible solutions for the [N II] $122 \mu \mathrm{m}$ emission are densities below $10^{3} \mathrm{~cm}^{-3}$ and $r_{\text {in }}$ higher than $10^{21.0} \mathrm{~cm}$. [Ne II] also agrees well with these conditions. At $T_{\text {eff }}$ of $35000 \mathrm{~K},[\mathrm{~S} \mathrm{III}]$ also emits, but remains below its observed value. Very little [N III], [Ne III], [O III], and [S IV] are produced 


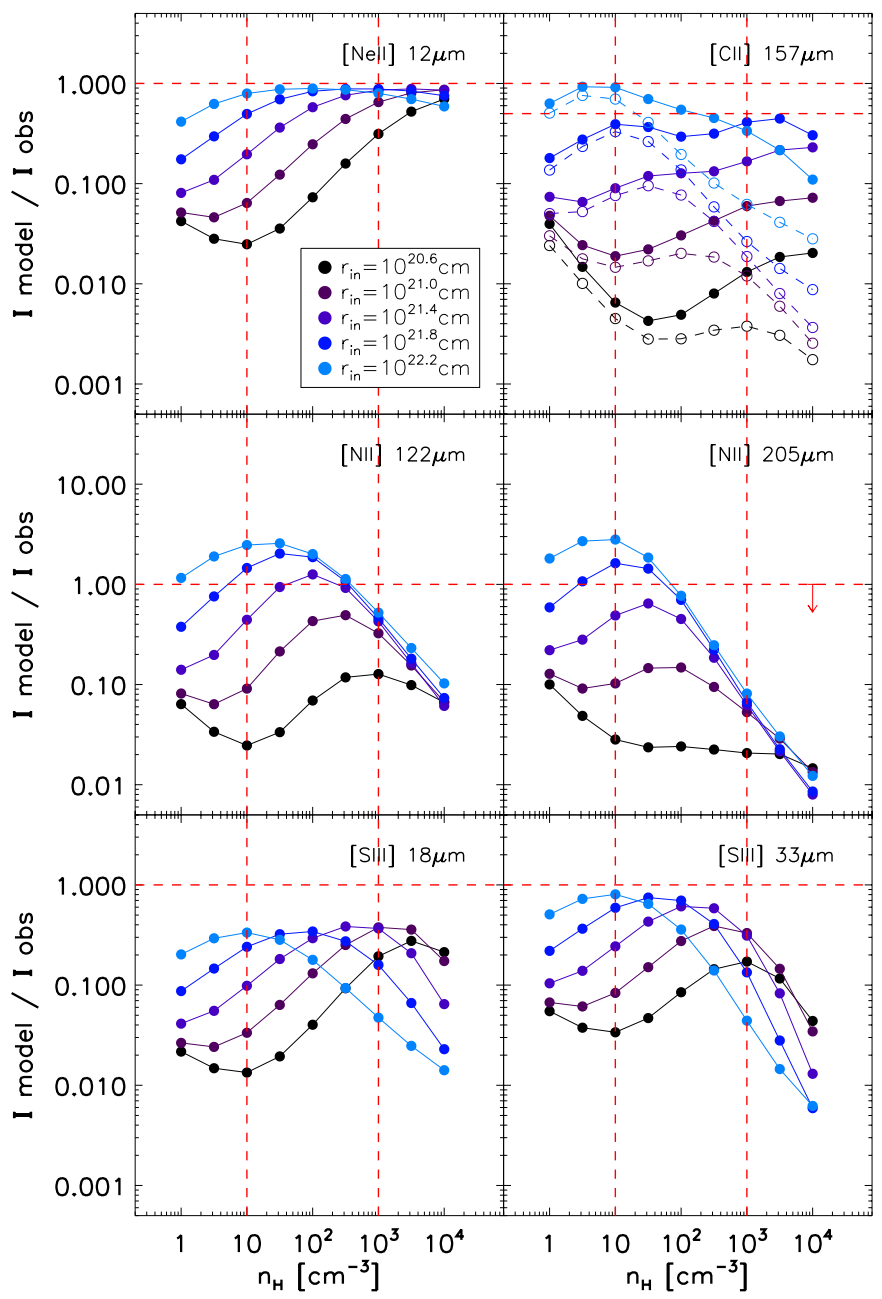

Fig. 9. Predictions for the model $\mathscr{D}$ of the $[\mathrm{Ne}$ II], [C II], [N II], and [S III] line intensities $\left(I_{\text {model }}\right)$, normalised by their observed value $\left(I_{\mathrm{obs}}\right)$ as a function of density $n_{\mathrm{H}}$. Models are for a diffuse ionised/neutral gas, with constant density, stellar temperature of $35000 \mathrm{~K}$, and are stopped when molecules start to form. The [S III], [Ne II] and [N II] lines emit in the ionised phase. The contribution of the ionised phase to the [C II] line is indicated with open circles. The vertical dashed lines indicate density values of the diffuse models $\mathscr{D}_{1}\left(n_{\mathrm{H}}=10 \mathrm{~cm}^{-3}\right)$ and $\mathscr{D}_{\mathrm{h}}\left(n_{\mathrm{H}}=10^{3} \mathrm{~cm}^{-3}\right)$ defined in Sect. 5.3. (This figure is available in color in electronic form.)

in this diffuse component, and no [O IV]. Although [N II] $122 \mu \mathrm{m}$ has a relatively low critical density, the density of the $\mathrm{N}^{+}$emitting medium is usually well constrained by the [N II] $205 \mu \mathrm{m}$ line. Unfortunately, the [N II] $205 \mu \mathrm{m}$ line is difficult to observe with PACS because of spectral leakage (see Sect. 2.2). The upper limit on the [N II] $205 \mu \mathrm{m}$ line sets an upper limit on the ratio [N II] $205 / 122 \mu \mathrm{m}$ of 0.75 which corresponds to a lower limit on the density of the "diffuse" gas component of $10 \mathrm{~cm}^{-3}$ (e.g. Rubin et al. 1994; Oberst et al. 2006). The [N II] $122 \mu \mathrm{m}$ line critical density sets an upper limit of $10^{3} \mathrm{~cm}^{-3}$. Models with densities between 10 and $10^{3} \mathrm{~cm}^{-3}$ work equally well for the "diffuse" medium in accounting for the [N II] $122 \mu \mathrm{m}$ and [Ne II] emission.

\section{3. [C II] contribution from the model $\mathscr{D}$}

Since $\mathscr{C}_{\text {PDR }}$ predicts very little [C II] $157 \mu \mathrm{m}$, we expect a significant contribution of the diffuse medium to the $\mathrm{C}^{+}$emission. $\mathrm{C}^{+}$is usually found in the surface layers of far-UV illuminated PDRs (Stacey et al. 1991; Negishi et al. 2001), but it can also come from the ionised gas, with a contribution up to $50 \%$ (Madden et al. 1993; Carral et al. 1994; Heiles 1994; Abel et al. 2005). With ionisation potentials lower than that of hydrogen, both $\mathrm{C}^{+}$ and $\mathrm{Si}^{+}$can be produced by low energy photons or excited by collisions with electrons or hydrogen atoms, depending on the degree of ionisation from the emitting medium.

Figure 9 shows the predicted [C II] line intensities from the diffuse medium with a stellar temperature of $35000 \mathrm{~K}$, as a function of gas density. The contribution of the diffuse phase (ionised+neutral) is in filled circles, and the contribution of the ionised phase only shown by open circles. Calculations are stopped when molecules start to form and the fraction of $\mathrm{H}_{2} / \mathrm{H}$ reaches $10 \%$. At low $n_{\mathrm{H}}$, the empty and filled circles are very close: a large fraction of the [C II] can arise from the ionised phase, until [C II] is collisionally de-excited by $\mathrm{e}^{-}\left(n_{\text {crit }}=\right.$ $50 \mathrm{~cm}^{-3}$ ), and emits mostly in the neutral phase, its intensity then increasing with density. At $r_{\text {in }}=10^{22.2} \mathrm{~cm}$, the [C II] predictions decrease for $n_{\mathrm{H}}>10^{2} \mathrm{~cm}^{-3}$, because the atomic phase is very thin as the material is cold and enters quickly into the molecular phase where the models stop. We disregard these models which do not predict enough [C II]. The range of possible solutions for the [C II] $157 \mu \mathrm{m}$ emission are $r_{\text {in }}$ higher than $10^{21.4} \mathrm{~cm}$, and are degenerate in $n_{\mathrm{H}}$. We can set a low-density case where $n_{\mathrm{H}}=10 \mathrm{~cm}^{-3}-$ for example. Then $r_{\text {in }}=10^{21.8} \mathrm{~cm}$ is the best fit, and [C II] comes mostly from the ionised phase, accounting for $\sim 45 \%$ of the observed value. We also consider a highdensity case where $n_{\mathrm{H}}=10^{3} \mathrm{~cm}^{-3}$, and $r_{\text {in }}=10^{21.8} \mathrm{~cm}$. In this case, $[\mathrm{C} \mathrm{II}]$ is mostly emitted in the neutral phase, accounting for $\sim 40 \%$ of the observed value. Both models fill a larger volume than the compact model $\mathscr{C}$.

\subsubsection{Constraint from the $\mathrm{H} \mid 21-\mathrm{cm}$ line}

The H I 21-cm mass upper limit of $10^{8} M_{\odot}$ from Bergvall et al. (2000) is an important gas diagnostic of the diffuse atomic gas and brings a strong constraint that we can use to differentiate between the low-density and high-density case models. The H I mass from $\mathscr{C}_{\text {PDR }}$ is $10^{7} M_{\odot}$. While the models from Fig. 9 were stopped at a fraction of $\mathrm{H}_{2} / \mathrm{H}$ reaching $10 \%$ to compute entirely the ionised and atomic phases, most of those models exceed the H I mass upper limit. By stopping those models when the upper limit on the H I mass is reached, we find that the lowdensity case model contribution to the [C II] line is unchanged since it comes from the ionised phase, while the high-density case model contributes to only $\sim 20 \%$ of the [C II] line intensity, since the atomic phase is stopped at lower $A_{\mathrm{V}}$ than previously computed.

For the rest of this study, we refer to the low-density case model as $\mathscr{D}_{1}$, and to the high-density case model as $\mathscr{D}_{\mathrm{h}}$. We cannot exclude the presence of a diffuse neutral gas, but in all cases, there is a prominent contribution to the [C II] from the ionised gas. We estimate that $\sim 40 \%$ originates in $\mathscr{D}_{1}$; the rest coming from $\mathscr{C}_{\text {PDR }}$ and little from $\mathscr{D}_{\mathrm{h}}$. Moreover, the diffuse models do not contribute more than $15 \%$ to the [O I] lines, which are reliable tracers of the PDR.

\section{Influence from other possible components}

\subsection{Magnetic fields}

Magnetic fields impact the model solution, dominating the pressure deep into the cloud, and they are expected to be important in star-forming regions (Shaw et al. 2009). Robishaw et al. (2008) found magnetic fields on the order of a few $\mathrm{mG}$ in ULIRGs, from $\mathrm{OH}$ Zeeman splitting. In local dwarf irregular galaxies, magnetic 
fields are found to be weak, of a few $\mu \mathrm{G}$ to $50 \mu \mathrm{G}$ in more extreme cases (e.g. NGC 1569, NGC 4214; Chyży et al. 2011; Kepley et al. 2010, 2011). No study on magnetic fields has been conducted for Haro 11, so we did not include them in our grid calculation. Nevertheless, in view of the fact that we have found that the density of the dense PDR plays a determining role in the predicted line fluxes, we investigate the influence of adding a magnetic field $B_{\mathrm{mag}}$ of strength $1 \mu \mathrm{G}$ to $3 \mathrm{mG}$ to the solution model $\mathscr{C}$ described in Sect 4.

The magnetic field $\left(B_{\text {mag }}\right)$ is expressed as a pressure term $\left(P_{\text {mag }}\right)$ through the cloud by the following equation:

$P_{\mathrm{mag}}=\frac{B_{\mathrm{mag}}^{2}}{8 \pi}$ and $B_{\mathrm{mag}}(r)=B_{\mathrm{mag}}(0)\left(\frac{n_{\mathrm{H}}(r)}{n_{\mathrm{H}}(0)}\right)^{\kappa}$, where $\kappa=2 / 3$

where $r$ is the depth of the cloud, $B_{\text {mag }}(0)$ and $n_{\mathrm{H}}(0)$ are the initial values of the magnetic field and hydrogen density. This density-magnetic field relationship from Crutcher (1999) corresponds to the case where gravity dominates over magnetic support in the cloud collapse, and the magnetic flux is conserved. Figure 10 shows the predicted [C II] and [O I] intensities with increasing $B_{\text {mag }}$. For low values of $B_{\text {mag }}$, thermal pressure, expressed as $P_{\text {gas }} \propto n_{\mathrm{H}} T_{\text {gas }}$, dominates and the densities in the PDR are high, with strong emission of [O I], as seen previously. With increasing $B_{\mathrm{mag}}$, magnetic pressure starts to dominate over gas pressure and the transition into the PDR is smooth, resulting in lower densities in the PDR, and less emission of [O I]. The [C II]/[O I] ratio increases with increasing $B_{\text {mag. The }}$ [C II] prediction falls between a factor of 0.5 and 1 of its observed value for all models. Values of $B_{\text {mag }}$ up to $10^{-3.75} \mathrm{G}$ predict enough [O I] emission. For $B_{\text {mag }}>10^{-3.75} \mathrm{G}$, the models under-predict the intensity of the PDR lines, which would require extra input power, although we are still within the errors (factor of 2).

However, when lowering the density, the size of the PDR layer increases, and the HI mass as well. With no magnetic field, we find $M(\mathrm{HI})=10^{7} M_{\odot}$ with a covering factor of $\sim 10 \%$. For $B_{\text {mag }}>10^{-4} \mathrm{G}$, we find $M(\mathrm{HI})>2 \times 10^{8} M_{\odot}$ for a covering factor of unity. By increasing the magnetic field strength, we go from a picture of small dense clumps to a more diffuse extended medium, which again does not agree with the observed upper limit on the H I mass. Moreover, high field strengths $(>100 \mu \mathrm{G})$ may be found in the cores of star formation, but are unlikely to hold on galaxy-wide scales. Models with $B_{\text {mag }}>10^{-4} \mathrm{G}$ are therefore discarded and appear in the shaded grey area in Fig. 10. The model with $B_{\text {mag }}$ of $10^{-4} \mathrm{G}$ is noted $\mathscr{C}_{B}$. Then, if we consider that all of the [O I] $145 \mu \mathrm{m}$ emission originates in the PDR, we can consequently scale the [C II] intensity and we estimate the contribution of the PDR to the [C II] line to be from $10 \%$ up to at most $50 \%$ in strong magnetic field cases.

\subsection{Origin of the $[\mathrm{O} I I 1] 88 \mu \mathrm{m}$ line}

Although emission from the [O III] $88 \mu \mathrm{m}$ line is expected in starburst galaxies, recent Herschel observations show that it is exceptionally bright in dwarf galaxies (Madden et al., in prep.). In Haro 11, it is the brightest of all MIR and FIR lines. With excitation potential of $35.1 \mathrm{eV}$ and critical density, $n_{\text {crit }}=5 \times$ $10^{2} \mathrm{~cm}^{-3}$, this line is expected to originate from a high ionisation and relatively low density medium. Effective temperatures, $T_{\text {eff }}>40000 \mathrm{~K}$, are usually needed to explain the bright [O III] emission in star-forming irregular galaxies (Hunter et al. 2001). When considering both models $\mathscr{C}_{\text {HII }}$ and $\mathscr{D}$, we find a good match between model predictions and observations for all

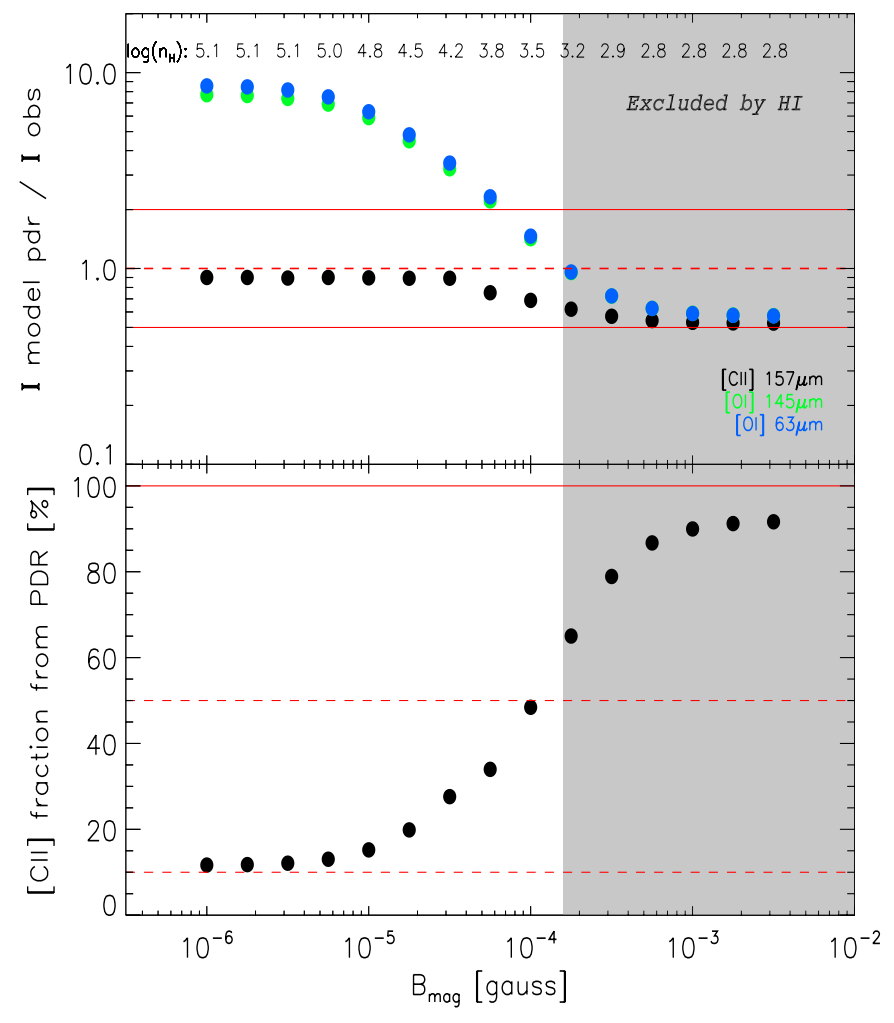

Fig. 10. Influence of the magnetic field on the PDR lines. Top: model predictions of the [C II] and [O I] intensities $\left(I_{\text {model }}\right)$ normalised by their observed value $\left(I_{\mathrm{obs}}\right)$ as a function of magnetic strength $B_{\mathrm{mag}}$. The effective density in the PDR is indicated for each $B_{\mathrm{mag}}$ value. Bottom: fraction of [C II] coming from the PDR, scaled to the [O I] $145 \mu \mathrm{m}$ prediction, assuming that all of it originates in the PDR. Models in the shaded area are not valid with respect to the upper limit on the H I column density. (This figure is available in color in electronic form.)

ionic lines considered, although the [O III] $88 \mu \mathrm{m}$ line is underpredicted by the models by a factor of $\sim 2$. $\mathscr{C}_{\text {HII }}$ accounts for $60 \%$ of the [O III] $88 \mu \mathrm{m}$ emission, and $\mathscr{D}$ does not predict any [O III] emission; the ionising photons are not energetic enough. This factor of 2 is within the uncertainties of this study, however we discuss the possible origin of the [O III] $88 \mu \mathrm{m}$ line from a highly ionised more diffuse medium. Although the [O III] emission is explained by $\mathscr{C}_{\mathrm{HII}}$ in the extreme case of Haro 11 , this may not be the case in other metal-poor dwarf galaxies.

In the star-forming region N11-B of the Large Magellanic Cloud, Lebouteiller et al. (2012) find bright [O III] $88 \mu \mathrm{m}$ emission that originates from spatially extended high-excitation diffuse ionised gas, and can be modeled by $\mathrm{O}$ stars distributed across the region. In the case of Haro 11 , the density in $\mathscr{C}_{\mathrm{HII}}$ is too high to fully reproduce the observed [O III] $88 \mu \mathrm{m}$ emission. As shown in Fig. 6, lowering the density in $\mathscr{C}_{\text {HII }}$ to densities $\leq 10^{2} \mathrm{~cm}^{-3}$ would better reproduce the observed [O III] $88 \mu$ m intensity.

Given the two components that we have modeled $\mathscr{C}_{\mathrm{HII}}$ and $\mathscr{D}$, we may consider a picture in which $\mathscr{C}_{\mathrm{HII}}$ does not have a covering factor of unity, but is actually porous. This would allow some fraction of the ionising photons to escape the compact H II region and travel further away in a lower density medium. Such a configuration could explain the [O III] $88 \mu \mathrm{m}$ emission, but would also lead to over-predicting several lines. In particular, in our case, predicted intensities of the [S IV], [O IV], and [N III] lines would be a factor of $\sim 2$ higher than their observed values. 

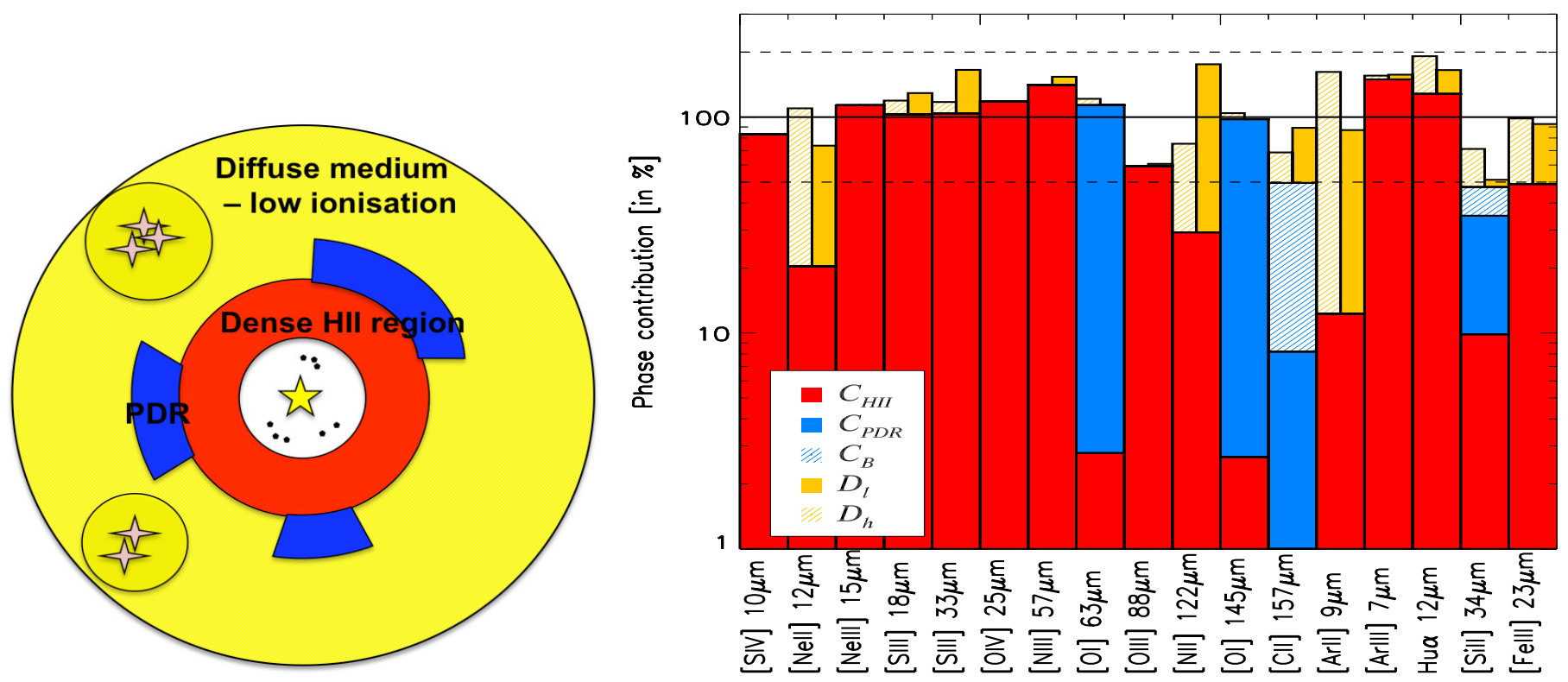

Fig. 11. Left: multi-phase build-up of the ISM of Haro 11, composed of a compact H II region $\left(\mathscr{C}_{\text {HII }}\right)$ in red, a dense fragmented PDR $\left(\mathscr{C}_{\text {PDR }}\right)$ in blue, a diffuse medium of lower ionisation $(\mathscr{D})$ in yellow, and warm dust in the inner region (represented as dots close to the starburst). Right: diagram of the contribution of each individual ISM phase to the global emission of the MIR and FIR lines. Red color corresponds to $\mathscr{C}_{\text {HII }}$, blue to $\mathscr{C}_{\text {PDR }}$, with possible enhancement in the [C II] (and [Si II]) lines when considering magnetic fields (hashed blue, $\mathscr{C}_{B}$ ), and yellow from the diffuse ionised medium $\left(\mathscr{D}_{1}\right)$, also with possible contribution from a diffuse neutral phase (hashed yellow, $\mathscr{D}_{\mathrm{h}}$ ). (This figure is available in color in electronic form.)

\subsection{Origin of the $[O I V]$ line and $X$-rays}

With an ionisation potential of $54.9 \mathrm{eV}$, [O IV] can only be excited by high energy sources. Its origin has been discussed in Lutz et al. (1998) or Schaerer \& Stasińska (1999). It can come from AGN activity (X-rays), very hot sources or shocks. In this Section, we explore the role of X-ray photons and/or shocks. In dwarf starbursting galaxies, a hot and young stellar population is usually responsible for the [O IV] emission. This has been confirmed by ISO observations of the dwarf galaxies II Zw 40 and NGC 5253 (Schaerer \& Stasińska 1999). Signatures of WolfRayet stars in the optical and P Cygni profiles in the far-UV $(\mathrm{O}$ VI lines), revealing the presence of $\mathrm{O}$ supergiants and a very young burst in Haro 11, were reported by Bergvall et al. (2006) and Grimes et al. (2007). In the case of Haro 11, we find that the young starburst containing Wolf-Rayet stars can indeed account for the [O IV] $25.9 \mu \mathrm{m}$ line intensity. The luminosity of the [O IV] line is $\sim 0.006 \%$ of $L_{\text {TIR }}$. Spinoglio et al. (2012) have compared the IR/submm emission lines to the IR luminosity in local samples of AGNs and starburst galaxies. They showed that the [O IV] line is weaker in starburst galaxies, about one order of magnitude less intense than in AGNs, and that starbursts follow

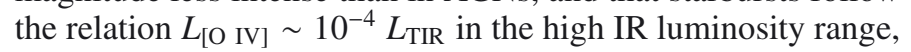
which is consistent with that observed in Haro 11. The [Ne V] $(97.1 \mathrm{eV})$ at $14.3 \mu \mathrm{m}$ is not detected in the Spitzer/IRS spectrum. The upper limit in Table 2 is consistent with our model prediction. Its absence confirms the fact that there is no AGN activity nor intense hard X-ray emission in Haro 11 . The X-ray component discussed in Sect. 3.3.1 in our model has little effect on the intensities of the ionic lines. Around the parameters of $\mathscr{C}_{\text {HII }}$, adding X-rays to the radiation field increases the intensity of the [O IV] $25.9 \mu \mathrm{m}$ line by only $\sim 1 \%$. In the PDR, the $\mathrm{X}$-rays have a moderate effect. They contribute up to $5 \%$ to the $[\mathrm{C} \mathrm{II}]$ intensity, and $\sim 10 \%$ to the $[\mathrm{O} \mathrm{I}]$ intensities. X-rays play a second order role and the physics of the neutral gas is dominated by the FUV photons (PDR), not the X-rays (XDR).

Shocks are also not our prefered explanation for the [O IV] emission. O'Halloran et al. (2008) find that the emission of the [Fe II] $26.0 \mu \mathrm{m}$ line in low-metallicity BCDs may be shock-derived or can result from a larger abundance of iron in the gas-phase as it is less depleted onto dust grains at low metallicity. However, the [Fe II] $26.0 \mu \mathrm{m}$ line is barely detected in Haro 11 . The observed ratio of $[\mathrm{Fe} \mathrm{III}] /[\mathrm{Fe}$ II] is $\sim 0.005$, which also tends to rule out shocks as a major heating process here.

\section{Multi-phase build-up of Haro 11}

We summarize the contributions of the different ISM phases that, when put together, account for the global MIR and FIR finestructure line emission observed in Haro 11 (Fig. 11). The panel on the left is an illustration of this multi-phase build-up: the central starburst is surrounded by our main model $\mathscr{C}$ composed of a compact $\mathrm{H}$ II region $\mathscr{C}_{\mathrm{HII}}$ (red), with adjacent dense PDRs $\mathscr{C}_{\text {PDR }}$ (blue). The volume around this compact nucleus is filled by diffuse ionised/neutral gas $\mathscr{D}$ (yellow). We also add a low filling factor component of warm dust close to the starburst (black dots), the need for which we discuss in Sect. 7.1. This scheme is the global picture (not to scale) that results from this modeling study. We remind the reader that the models $\mathscr{C}$ and $\mathscr{D}$ were computed separately. The panel on the right is a histogram indicating the contribution of each model to the line intensities, with identical color-coding. All 17 lines considered are reproduced by the 3 models $\mathscr{C}_{\text {HII }}, \mathscr{C}_{\text {PDR }}$, and $\mathscr{D}$ within a factor of 2 . The model parameters of these phases and line predictions are also listed in Table 5. In view of the results from Sects. 5.3 and 6.1, in which the upper limit on the H I mass limits the contribution from the diffuse neutral medium and from magnetic fields, the models $\mathscr{D}_{\mathrm{h}}$ and $\mathscr{C}_{B}$ are not taken into account for our final build-up of the ISM of Haro 11. The models we combine are:

- a compact H II region that dominates the emission of the ionic lines, $\mathscr{C}_{\mathrm{HII}}$

- a dense PDR of coverage $\sim 10 \%, \mathscr{C}_{\text {PDR }}$;

- a diffuse low-ionisation medium, $\mathscr{D}_{1}$.

We analyse results from this build-up in terms of dust emission, extinction, and mass budget. 
Table 5. Model parameters and line predictions.

\begin{tabular}{|c|c|c|c|c|c|c|}
\hline \multirow[t]{2}{*}{ Quantity } & \multicolumn{6}{|c|}{ Model } \\
\hline & $\mathscr{C}_{\text {HII }}+$ & $\mathscr{C}_{\mathrm{PDR}}$ & $\mathscr{D}_{1}$ & $\mathscr{D}_{\mathrm{h}}$ & Warm dust & Total $^{a}$ \\
\hline Energy source & Starburs & $3.7 \mathrm{Myr}$ & Star $35000 \mathrm{~K}$ & Star $35000 \mathrm{~K}$ & Starburst 3.7 Myr & \\
\hline$n_{\mathrm{H}}\left[\mathrm{cm}^{-3}\right]$ & $10^{2.8}$ & $10^{5.1}\left(10^{3.4}\right)$ & $10^{1.0}$ & $10^{3.0}$ & $10^{1.5}$ & \\
\hline$r_{\text {in }}[\mathrm{cm}]$ & $10^{21.4}$ & $10^{21.4}$ & $10^{21.8}$ & $10^{21.8}$ & $10^{19.8}$ & \\
\hline$I([\mathrm{~S}$ IV $] 10.5) / I_{\mathrm{obs}}{ }^{b}$ & $0.84(0.29)$ & - & - & - & - & 0.84 \\
\hline$I([\mathrm{Ne}$ II $] 12.8) / I_{\mathrm{obs}}$ & $0.20(0.10)$ & - & 0.53 & 0.89 & - & 0.73 \\
\hline$I([\mathrm{Ne} \mathrm{III}] 15.6) / I_{\mathrm{obs}}$ & $1.13(0.61)$ & - & - & - & - & 1.13 \\
\hline$I([\mathrm{~S}$ III $] 18.7) / I_{\mathrm{obs}}$ & $1.03(0.50)$ & - & 0.26 & 0.16 & - & 1.29 \\
\hline$I([\mathrm{~S}$ III $] 33.5) / I_{\mathrm{obs}}$ & $1.04(0.63)$ & - & 0.61 & 0.14 & - & 1.65 \\
\hline$I([\mathrm{Si}$ II $] 34.8) / I_{\mathrm{obs}}$ & $0.11(0.07)$ & $0.24(0.37)$ & 0.04 & 0.24 & - & 0.39 \\
\hline$I(\mathrm{Hu} \alpha 12.4) / I_{\mathrm{obs}}$ & $1.28(0.61)$ & - & 0.37 & 0.63 & - & 1.65 \\
\hline$I([\mathrm{Fe}$ III $] 22.9) / I_{\mathrm{obs}}$ & $0.49(0.27)$ & - & 0.44 & 0.50 & - & 0.93 \\
\hline$I([\mathrm{O}$ IV $] 25.9) / I_{\mathrm{obs}}$ & $1.18(0.74)$ & - & - & - & 0.10 & 1.28 \\
\hline$I([\mathrm{Ar} \mathrm{III}] \quad 8.99) / I_{\mathrm{obs}}$ & $1.49(0.51)$ & - & 0.08 & 0.06 & - & 1.57 \\
\hline$I([\mathrm{Ar}$ II $] 6.99) / I_{\mathrm{obs}}$ & $0.12(0.08)$ & - & 0.75 & 1.49 & - & 0.87 \\
\hline$I([\mathrm{C}$ II $] 157) / I_{\mathrm{obs}}$ & $0.01(0.01)$ & $0.07(0.49)$ & 0.40 & 0.19 & - & 0.48 \\
\hline$I([\mathrm{O} \mathrm{III}] 88) / I_{\mathrm{obs}}$ & $0.59(0.44)$ & - & 0.02 & - & - & 0.61 \\
\hline$I([\mathrm{O}$ I $] 63) / I_{\mathrm{obs}}$ & $0.03(0.02)$ & $1.11(1.03)$ & - & 0.08 & - & 1.14 \\
\hline$I([\mathrm{O} \mathrm{I}] 145) / I_{\mathrm{obs}}$ & $0.03(0.02)$ & $0.95(1.00)$ & - & 0.07 & - & 0.98 \\
\hline$I([\mathrm{~N} \mathrm{III]}] 57) / I_{\mathrm{obs}}$ & $1.41(0.99)$ & - & 0.13 & - & - & 1.44 \\
\hline$I([\mathrm{~N} \mathrm{II}] 122) / I_{\mathrm{obs}}$ & $0.29(0.23)$ & - & 1.46 & 0.46 & - & 1.75 \\
\hline
\end{tabular}

Notes. ${ }^{(a)}$ The total is taken as the sum of models $\mathscr{C}, \mathscr{D}_{1}$ and warm dust, which is what we consider for the final build-up in Sect. 7; although $\mathscr{D}_{\mathrm{h}}$ and $\mathscr{C}_{B}$ may also alter the line predictions. ${ }^{(b)}$ Ratio of the predicted intensity of the model over the observed intensity $\left(I_{\mathrm{obs}}\right)$. Ratio values below $1 \%$ are not indicated.

\subsection{The global SED}

The proposed geometry also implies a prediction for the dust emission coming from the different ISM phases of Haro 11. We compare the predicted SED of the multi-phase build-up with the observations of Haro 11 in Fig. 12. The goal here is not to fit in detail the dust emission but rather to reproduce the global shape of the SED because it is sensitive to the temperature of the dust, which is one of the main predictions of the multi-phase model. The SED of the compact model $\mathscr{C}$ (both $\mathscr{C}_{\text {HII }}$ and $\mathscr{C}_{\text {PDR }}$ ) peaks at $\sim 40 \mu \mathrm{m}$ (Fig. 12), as in the observed SED. In particular, $\mathscr{C}_{\mathrm{PDR}}$ is too dense to explain the FIR shape of the SED, which corroborates the presence of a more diffuse component. Indeed, the diffuse component $\mathscr{D}_{1}$ matches the temperature of Haro 11 in the FIR, although the longest wavelengths, beyond $250 \mu \mathrm{m}$, are still under-predicted. Perhaps the most surprising result is that the multi-phase model fails completely to reproduce the UV-optical extinction and the MIR emission, despite the choice of a lower cut in the grain sizes (Sect. 3.3.3). We discuss these two points in Sects. 7.1.1 and 7.1.2.

\subsubsection{Energy balance}

In the UV-optical wavelength range, the total outward luminosity (Fig. 12: bottom panel, black line) is $80 \%$ greater than the observed luminosity. Has the input energy in the UV-optical wavelength range been over-estimated? We are confident about the shape of the input starburst spectrum since the spectral line ratios depend on the hardness of the radiation field and are well fitted by our models. Additional confidence draws from the right amount of input energy as the level of the NIR photometry, unaffected by the little extinction present in Haro 11, is well matched.

The SED reflects the global energy balance of Haro 11 and shows that a fraction of the total input energy injected in the UV-optical wavelength range is not absorbed. The models $\mathscr{C}_{\text {HII }}$ and $\mathscr{D}_{1}$ are responsible for the over-prediction in the
UV-optical. We find the average extinction of the model to be $A_{\mathrm{V}}=0.15 \mathrm{mag}$. The spectrum is dominated by emission from the ionised model components: $\mathscr{C}_{\mathrm{HII}}$, of which $90 \%$ of the radiation escapes since the PDR covers only $10 \%$, and $\mathscr{D}_{1}$. These models are computed separately and work as MIR-FIR emission line diagnostics, very little affected by dust attenuation, but we neglect their interaction. We can estimate the reddening needed to match the level of the model spectrum to the observed data points. Using the extinction law of Calzetti et al. (2000), with $R_{\mathrm{V}}=3.3$ set by the model, we find $A_{\mathrm{V}}=0.9$ mag, hence $A_{B}=1.2 \mathrm{mag}$ and $E(B-V)=0.3 \mathrm{mag}$. The reddened spectrum appears in dashed dark line in Fig. 12 (bottom). From UV continuum measurements and $\mathrm{H} \alpha / \mathrm{H} \beta$ ratio, Bergvall $\&$ Östlin (2002) estimate $A_{B}=0.7 \mathrm{mag}$. The optical image (Fig. 1) shows a dust lane passing in front of knot B, where the extinction is known to be higher (Adamo et al. 2010; Guseva et al. 2012). Adamo et al. (2010) find that $E(B-V)$ is 0.4 towards knot B and 0.1 towards knot $\mathrm{C}$.

On the contrary, in the MIR (5 to $40 \mu \mathrm{m}$ ), the predicted luminosity is two times lower than the observed luminosity. This emission in the MIR can be attributed to warm dust that we model in the following section.

This suggests an interpretation in terms of geometry of the galaxy, in which a large number of ionising photons can travel far, but in the end, the different media (compact and diffuse) are well mixed together, yielding a significant dust column along most lines of sight. In particular, the warm dust component that we add close to the central starburst and emits in the MIR is likely responsible for the absorption of the UV-optical part of the $\mathscr{C}_{\text {HII }}$ spectrum which is the primary contributor to the total spectrum in this range.

\subsubsection{Warm dust component}

To account for the observed warm dust that our models do not produce in the MIR range (Fig. 12, top panel), we have examined 

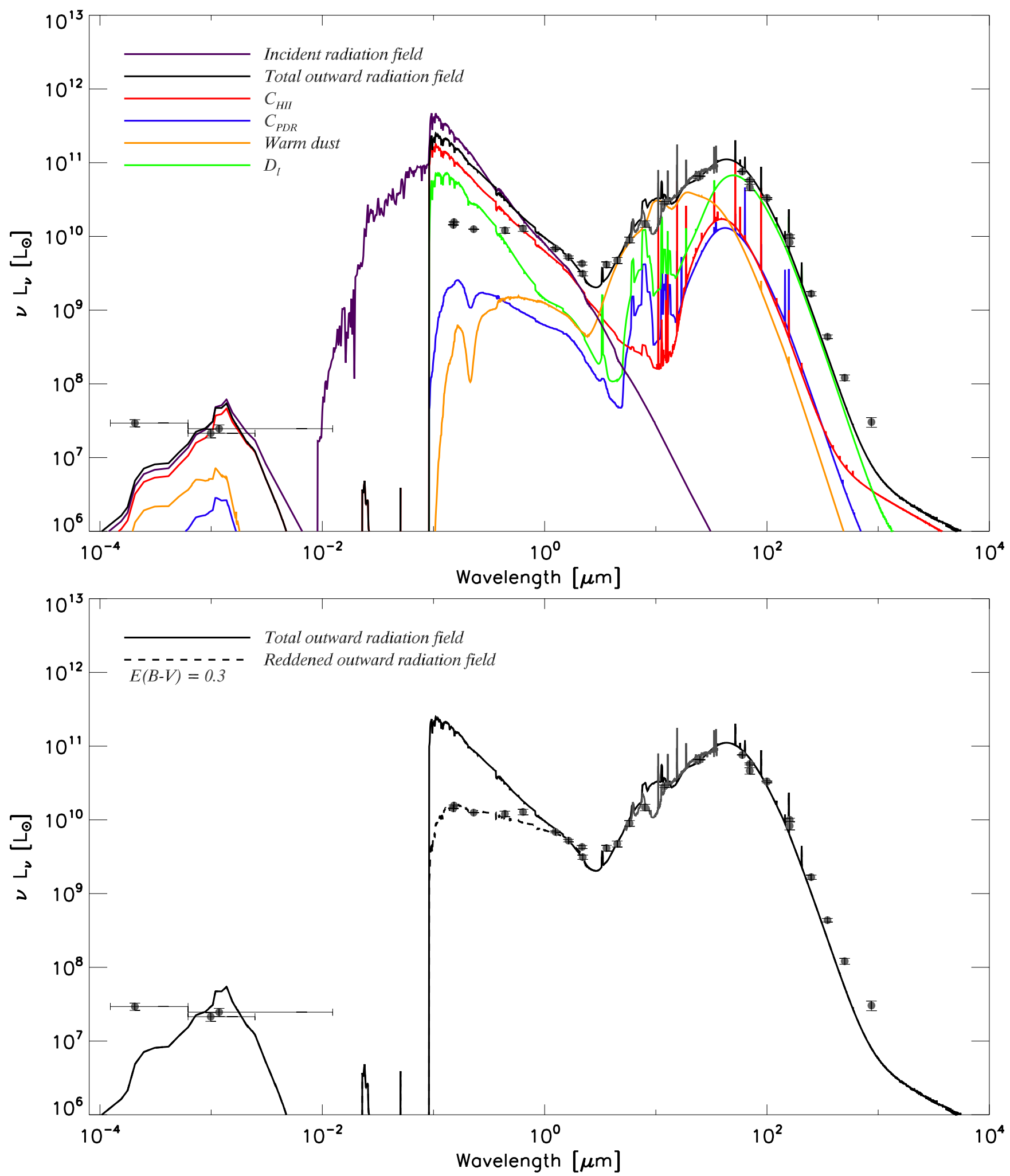

Fig. 12. Top: spectral energy distribution (SED) of the incident radiation field striking the cloud and outward radiation from each component modeled by Cloudy. The dark spectrum is the total outward radiation resulting from multi-phase build-up, and arriving to the observer. Photometry data from NED, Bendo et al. (2012), Galametz et al. (2009), and Rémy et al. (in prep.), and the IRS spectrum are overlaid in grey. Bottom: spectral energy distribution of the total outward radiation field and the reddened outward radiation field in dashed dark line. (This figure is available in color in electronic form.)

two possible solutions that do not change the intrinsic properties of the dust grains (which are little known):

1) change the dust distribution for a postshock distribution (Jones et al. 1996), where grains are affected by shocks, with preferential destruction of large grains, resulting in an enhancement of small grains with respect to large grains. This grain shattering results in a distribution with steeper slope and lower cut-off sizes. This distribution is successfully 
applied to model IR SEDs of LIRGs in Dopita et al. (2011). Galliano et al. (2003) used this distribution, that may have been generated by supernova shocks, to model the dust in the dwarf galaxy NGC 1569. However we have been unable to satisfactorily fit the SED by changing the grain properties;

2) add a warm dust component close to the central starburst. In this way we add to our picture a porous layer of dust particles close to the central starburst, which is not inconceivable for compact H II regions. Models from Groves et al. (2008) show that the observed warm dust emission arises from hot dust embedded within the H II region. This is also supported by spatially resolved observations of nearby galaxies. Calzetti et al. (2005) find that the $8 \mu \mathrm{m}$ and $24 \mu \mathrm{m}$ emission originate within the $\mathrm{H}$ II regions.

We adopt a warm dust component, at a distance of $r_{\text {in }}=$ $10^{19.8} \mathrm{~cm}$, with density $n_{\mathrm{H}}=10^{1.5} \mathrm{~cm}^{-3}$ and covering factor $15 \%$ (orange spectrum in Fig. 12). These parameters are chosen to reproduce the level of the SED in the MIR. In these conditions grains can easily survive. This component has little or no effect on the discussed emission lines because of its compactness. The photons are predominantly absorbed by the dust, not the gas, and dust collisions are responsible for the cooling. This warm dust component may be regarded as an equivalent of an ultra-compact H II region (Dopita et al. 2003), or the hot spots described in Siebenmorgen \& Krügel (2007). This component takes away $15 \%$ of the input luminosity from the H II region model. The amount of necessary energy and the hardness of the radiation field can be adjusted by modifying simultaneously the input luminosity, covering factor, and the inner radius in the code. Integrating the spectrum from 3 to $1100 \mu \mathrm{m}$ gives $L_{\mathrm{TIR}}=1.7 \times 10^{11} L_{\odot}$, very close to the infered dust-model value of $1.4 \times 10^{11} L_{\odot}$ from Galametz et al. (2009) and Rémy et al. (in prep.).

This warm dust model matches the continuum level in the NIR-MIR, but produces a silicate feature in emission at $9.7 \mu \mathrm{m}$, which is not seen in the Spitzer/IRS spectrum. This discrepancy is likely due to the fact that we have modeled the compact and diffuse phases of the ISM independently. Foreground dust from the other components may in turn extinguish the silicate seen in emission. Another factor at play is the adopted abundance of silicate grains. Quantitative surveys with Spitzer of AGB carbon and silicate dust production rates in the Magellanic Clouds find that carbon dust injection rates dominate over those of silicate (Matsuura et al. 2009; Boyer et al. 2012). There may be a small effect from foreground cold dust from the molecular phase of Haro 11 that we do not model, which would have low filling factor and high $A_{\mathrm{V}}$.

\subsubsection{FIR-mm wavelength range}

The mismatch between the observed and predicted SED beyond $250 \mu \mathrm{m}$ (Fig. 12) may be attributed to colder dust in the molecular phase. With no molecular gas constraint, we cannot include a cold molecular phase in our modeling. However, as a first approximation on the required dust properties, we can continue $\mathscr{C}_{\mathrm{PDR}}$ to higher $A_{\mathrm{V}}$ and stop the model when it matches the submm temperature. This way, we estimate that the missing emission requires high $A_{\mathrm{V}} \sim 40 \mathrm{mag}$ and agrees with a grain temperature of $\sim 25 \mathrm{~K}$, which would fit the SPIRE data to $500 \mu \mathrm{m}$ but still under-predict the LABOCA $870 \mu \mathrm{m}$ data (see Fig. A.1 in Appendix A). The latter is rather related to the submm excess introduced in Sect. 1.1. This excess is modeled by a cold dust component in Galametz et al. (2009).
Table 6. Optical line predictions.

\begin{tabular}{|c|c|c|c|c|}
\hline \multirow[t]{2}{*}{ Line } & \multicolumn{4}{|c|}{$I / I(\mathrm{H} \beta)$} \\
\hline & Observations $^{a}$ & $\mathscr{C}_{\text {HII }}$ & $\mathscr{D}_{1}=$ & Total model $^{b}$ \\
\hline [O II $] 3727 \AA$ & 2.3 & 1.4 & 3.5 & 2.3 \\
\hline $\mathrm{H} \beta 4861 \AA$ & 1.0 & 1.0 & 1.0 & 1.0 \\
\hline [O III] $4959 \AA$ & 1.1 & 1.7 & 0.08 & 1.5 \\
\hline [O III] $5007 \AA$ & 3.4 & 5.2 & 0.03 & 4.5 \\
\hline $\mathrm{H} \alpha 6563 \AA$ & 2.9 & 2.6 & 2.0 & 2.9 \\
\hline [N II] $6583 \AA$ & 0.53 & 0.32 & 0.79 & 0.52 \\
\hline [S II] $6716 \AA$ & 0.22 & 0.07 & 0.03 & 0.07 \\
\hline [S II] $6731 \AA$ & 0.12 & 0.08 & 0.02 & 0.08 \\
\hline [S III] $9069 \AA$ & 0.15 & 0.17 & 0.13 & 0.19 \\
\hline [S III] $9532 \AA$ & 0.41 & 0.42 & 0.32 & 0.47 \\
\hline
\end{tabular}

Notes. ${ }^{(a)}$ Ratio of the observed line intensity over $\mathrm{H} \beta$ from Guseva et al. (2012). The fluxes are extinction-corrected and averaged over the two knots B and C. ${ }^{(b)}$ Ratio of the intrinsic line intensity over $\mathrm{H} \beta$ predicted by Cloudy.

\subsection{Optical lines}

Several optical lines, including $\mathrm{H} \alpha, \mathrm{H} \beta$, [O III $] 5007 \AA$, have been observed towards the central visual peak in Bergvall \& Östlin (2002). Because of the small aperture of the observations, $4^{\prime \prime} \times 4^{\prime \prime}$, an absolute flux comparison cannot be made, except for $\mathrm{H} \alpha$ which was fully mapped in Östlin et al. (1999) with the ESO $3.6 \mathrm{~m}$ telescope. More recent observations were also performed with the VLT/X-shooter, in the wavelength range 3000-24000 $\AA$, by Guseva et al. (2012). In particular, they observed, in a slit of size $\sim 1^{\prime \prime} \times 11^{\prime \prime}$, the $\mathrm{H} \alpha, \mathrm{H} \beta$, [O II] $3727 \AA$, [O III] 4959 and $5007 \AA$, [S II] 6716 and $6731 \AA$, [S III] 9069 and $9535 \AA$ lines, in both knots B and C of Haro 11. From these observations, they determined the metallicity of Haro 11 and elemental abundances. Optical lines are affected by dust extinction, and comparison of the model predictions to the observations is very dependent on the adopted geometry. With Cloudy, we can compare the intrinsic emission predicted by our model to the extinction-corrected observations.

The ratio of the optical lines listed above to $\mathrm{H} \beta$ are all well reproduced by our Cloudy models, within $40 \%$, with contribution from both the H II region and the diffuse medium (Table 6). The only line which poses a problem is [S II], which is overestimated by a factor of 3 at $6716 \AA$, and 1.5 at $6731 \AA$. The Cloudy ratio of the two [S II] lines is thus 0.9. The Cloudy models of Guseva et al. (2012) also over-predict the [S II] lines by a factor of 2, but reproduce their observed ratio of 1.5 . This ratio is sensitive to the electron density of the medium probed, which in their case is a density of $10 \mathrm{~cm}^{-3}$. We find that our diffuse ionised model with density $10 \mathrm{~cm}^{-3}$ does predict a [S II] line ratio of 1.5. Concerning $\mathrm{H} \alpha$, we compare its absolute flux and find that it emits from both the H II region and the diffuse medium, with a total predicted luminosity of $7 \times 10^{8} L_{\odot}$, close to the observed value of Östlin et al. (1999), $L_{\mathrm{H} \alpha}=8 \times 10^{8} L_{\odot}$.

\subsection{Mass budget}

The gas mass of each ISM component can be calculated using the density profile in the cloud and integrating over the volume of each slice inside the cloud. We then multiply the gas mass by 1.36 to account for helium, and use the $\mathrm{D} / \mathrm{G}$ mass ratio of the model $\left(2.64 \times 10^{-3}\right)$ to derive the dust mass. The masses of each component are summarized in Table 7 . We find a mass of 
Table 7. Mass budget in solar mass $M_{\odot}$.

\begin{tabular}{lccc}
\hline \hline Model & $M(\mathrm{H} \mathrm{II})$ & $M(\mathrm{PDR})^{a}$ & $M_{\text {dust }}$ \\
\hline $\mathscr{C}_{\text {HII }}$ & $3.0 \times 10^{7}$ & - & $7.2 \times 10^{4}$ \\
$\mathscr{C}_{\text {PDR }}$ & - & $1.2 \times 10^{8}$ & $3.2 \times 10^{5}$ \\
$\mathscr{D}_{1}$ & $5.5 \times 10^{8}$ & $1.2 \times 10^{8}$ & $1.7 \times 10^{6}$ \\
Warm dust & - & - & $8.5 \times 10^{3}$ \\
Total & $5.8 \times 10^{8}$ & $2.4 \times 10^{8}$ & $2.1 \times 10^{6}$ \\
Literature & $\sim 10^{9 b}$ & $2 \times 10^{8 c}$ & $\sim 6 \times 10^{6 d}$ \\
\hline
\end{tabular}

Notes. ${ }^{(a)} M(\mathrm{PDR})$ is the sum of the $\mathrm{HI}$ mass and the $\mathrm{H}_{2}$ mass in the PDR envelope untraced by CO. ${ }^{(b)}$ Bergvall \& Östlin (2002). ${ }^{\left({ }^{c}\right)}$ Bergvall et al. (2000), where they also report $M(\mathrm{H} \mathrm{I})<10^{8} M_{\odot} .{ }^{(d)}$ This is the dust mass without including the cold component of $10 \mathrm{~K}$ from Galametz et al. (2009). Their total dust mass is $2 \times 10^{7} M_{\odot}$.

ionised gas $\left(\mathscr{C}_{\mathrm{HII}}+\mathscr{D}_{1}\right)$ of $5.8 \times 10^{8} M_{\odot}$, and a mass of neutral gas $\left(\mathscr{C}_{\mathrm{PDR}}+\mathscr{D}_{1}\right)$ of $2.4 \times 10^{8} M_{\odot}$. This neutral gas mass accounts for the $\mathrm{HI}$ mass as well as the mass of $\mathrm{H}_{2}$ formed in PDR envelopes before the $\mathrm{CO}$ formation. This $\mathrm{H}_{2}$ layer not traced by $\mathrm{CO}$ is referred as the "dark gas" (Wolfire et al. 2010). The PDR- $\mathrm{H}_{2}$ mass is $\sim 10^{8} M_{\odot}$ and comes from $\mathscr{C}_{\mathrm{PDR}}$, while the H I mass comes essentially from the diffuse model $\mathscr{D}_{1}$ with $M(\mathrm{HI}) \sim 10^{8} M_{\odot}$ fixed by its upper limit. As expected, the ionised phase is dominant in Haro 11. The ionised gas and PDR masses are similar to that of Bergvall et al. (2000). We derive a total dust mass of $2.1 \times 10^{6} \mathrm{M}_{\odot}$, comparable to the value $6 \times 10^{6} \mathrm{M}_{\odot}$ of Galametz et al. (2009).

These dust and gas mass inventories do not include modeling of the cold CO-cloud. The missing FIR emission discussed in Sect. 7.1.3 results in a dust mass of $\sim 2 \times 10^{6} M_{\odot}$, which does not include the observed submm excess. When modeling the dust submm excess as cold dust, Galametz et al. (2009) find a total dust mass of $2 \times 10^{7} M_{\odot}$, which would result in a higher $\mathrm{D} / \mathrm{G}$ mass ratio $(\sim 1 / 50)$ compared to our current gas modeling. A significant fraction of ISM mass would therefore not be accounted for by our models, part of which should sit in the molecular phase that we have not modeled. $\mathrm{CO}(1-0)$ is to date undetected (Bergvall et al. 2000). The state of a reservoir of warm molecular gas is addressed in a forthcoming paper (Cormier et al., in prep.). We would like to emphasize that the determination of the cold dust mass is very sensitive to the model and geometry adopted. Both the cool dust interpretation of the submm excess and the model accounting for the missing FIR emission (Sect. 7.1.3) are, without further constraints on the cold phase, ad hoc means to fit the dust SED. In particular, the cold dust interpretation of the submm excess has little physical support as it is unlikely to encounter such very large columns of (cold shielded) dust in dwarf galaxies, and because the submm excess does not seem to correlate spatially with the densest ISM phases (Galliano et al. 2011).

\section{Conclusion}

We have presented Spitzer/IRS and Herschel/PACS spectroscopic observations of the MIR-FIR fine-structure cooling lines in the starburst low-metallicity galaxy Haro 11. Investigating the nature of the different gas phases in this galaxy, we have modeled the ISM phases of Haro 11 step by step with the spectral synthesis code Cloudy. The main results can be summarized as follows:

1. The IRS and PACS spectra show very bright MIR and FIR fine-structure cooling lines, the brightest of all being the [O III] $88 \mu \mathrm{m}$ line. The galaxy is undergoing young active star formation, as traced by the [O III] $88 \mu \mathrm{m}$ line $(35.1 \mathrm{eV})$, which is about 3 times brighter than the classical neutral gas tracers [C II] $157 \mu \mathrm{m}$ and [O I] $63 \mu \mathrm{m}$. High line ratios of $[\mathrm{Ne}$ III $] /[\mathrm{Ne}$ II $],[\mathrm{S} \mathrm{IV}] /[\mathrm{S} \mathrm{III]}]$, and $[\mathrm{N} \mathrm{III]}] /[\mathrm{N}$ II $]$ also trace the hard radiation field. We find $L_{[\mathrm{C} \text { II }]} / L_{\mathrm{TIR}}=0.1 \%$, $\left(L_{[\mathrm{C} \text { II }]}+L_{[\mathrm{O} \text { I] }}\right) / L_{\mathrm{TIR}}=0.2 \%$, and altogether, the MIR and FIR lines represent $1.2 \%$ of $L_{\mathrm{TIR}}$.

2. We model our exhaustive dataset of 17 fine-structure lines with Cloudy and find the need to describe Haro 11 with these component phases:

- a dense H II region, illuminated by a young starburst; from which most of the ionic lines originate;

- adjacent dense PDR of low $(\sim 10 \%)$ covering factor;

- an extended diffuse low-ionised/neutral medium;

- a porous warm dust component close to the stars that accounts for the elevated MIR continuum.

3. Emission from the [N II] $122 \mu \mathrm{m}$ and [Ne II] lines cannot be reconciled with the compact $\mathrm{H}$ II region but are rather associated with a more diffuse low-ionisation medium.

4. We find that $10 \%$ of the [C II] emission is associated with the dense PDR, and up to $50 \%$ when lowering the density. About $50 \%$ of the [C II] arises from a more diffuse ionised medium. The low extinction and low metallicity of Haro 11 make its ISM more leaky, and the emitting region of [C II] larger. The [O I] emission is fully associated with the PDR. The observed [C II] luminosity is comparable to that of the $[\mathrm{O} \mathrm{I}] 63 \mu \mathrm{m}$ line. In the dense component we derive $L_{[\mathrm{C} \text { II] }} / L_{\mathrm{TIR}} \sim 0.05 \%$ and $\left(L_{[\mathrm{C} \text { II] }}+L_{[\mathrm{O} \text { I] }}\right) / L_{\mathrm{TIR}} \sim 1 \%$, which is a common measure of the gas heating efficiency in the neutral gas, and in the diffuse component we find $L_{\text {[C II] }} / L_{\text {TIR }} \sim 0.1 \%$.

5. The only line that we do not completely reconcile with our model is the [O III] $88 \mu \mathrm{m}$. Our compact H II region model accounts for $50 \%$ of the emission of [O III]. Our prefered explanation for the intense [O III] emission is the presence of yet another additional diffuse component, filling the volume around the porous H II region, and where the gas is heated by escaping UV photons.

6. We estimate the mass from each modeled phase. We find a mass of ionised gas of $6 \times 10^{8} M_{\odot}$, a PDR mass of $2 \times 10^{8} M_{\odot}$, and a dust mass (without submm constraints, because of the submm excess) of $2 \times 10^{6} M_{\odot}$. The ionised gas mass is larger than the atomic gas mass.

7. Finally, in terms of structure, the ISM of Haro 11 appears to be mostly filled with extended diffuse gas. Our simple picture can reproduce the observations by a galaxy with a filling factor of diffuse neutral and ionised gas of at least $90 \%$, a dense $\mathrm{H}$ II region component of filling factor $\sim 0.2 \%$, and a PDR component of filling factor $<0.01 \%$. A radiative transfer model which takes into account clumpy source and gas structures would provide a more realistic picture of Haro 11.

Acknowledgements. The authors would like to thank Albrecht Poglitsch and Alessandra Contursi for their help with the PACS data. Part of this work has been made possible by financial support from the CNRS/INSU programme PCMI. PACS has been developed by a consortium of institutes led by MPE (Germany) and including UVIE (Austria); KU Leuven, CSL, IMEC (Belgium); CEA, LAM (France); MPIA (Germany); INAF-IFSI/OAA/OAP/OAT, LENS, SISSA (Italy); IAC (Spain). This development has been supported by the funding agencies BMVIT (Austria), ESA-PRODEX (Belgium), CEA/CNES (France), DLR (Germany), ASI/INAF (Italy), and CICYT/MCYT (Spain). This research has made use of the NASA/IPAC Extragalactic Database (NED) which is operated by the Jet Propulsion Laboratory, California Institute of Technology, under contract with the National Aeronautics and Space Administration. Based on observations made with the NASA/ESA Hubble Space Telescope, and obtained from the Hubble Legacy Archive, which is a collaboration between the Space Telescope Science Institute (STScI/NASA), the Space Telescope European Coordinating Facility (ST-ECF/ESA) and the Canadian Astronomy Data Centre (CADC/NRC/CSA). 


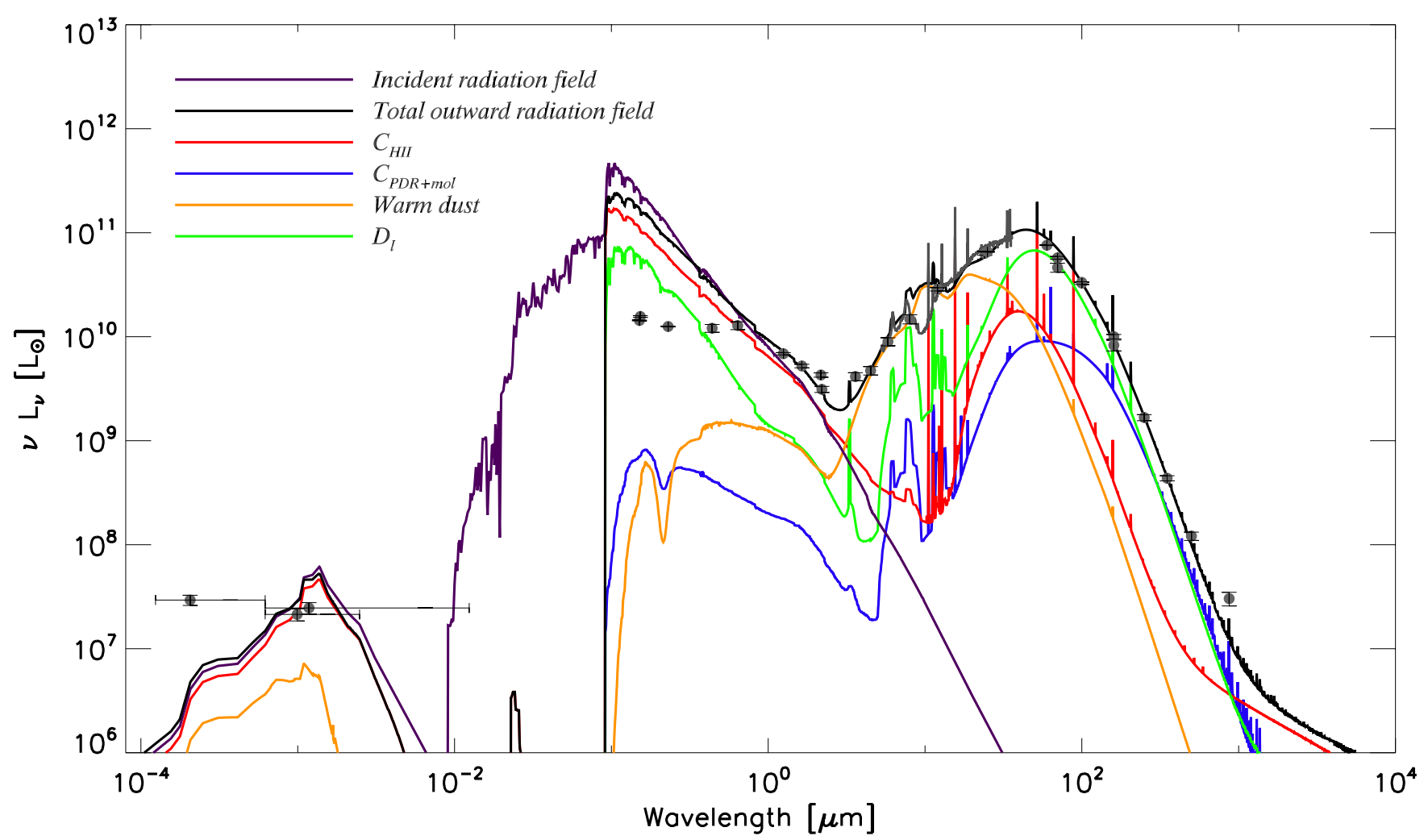

Fig. A.1. Same as Fig. 12 (top panel) except that the PDR component is continued to higher $A_{\mathrm{V}}$ in the molecular regime to match the observed submm photometry data. The covering factor of this molecular component is of $\sim 5 \%$. (This figure is available in color in electronic form.)

\section{Appendix A: SED including the cold molecular component}

We model the SED beyond $250 \mu \mathrm{m}$ by stopping the dense PDR model $\mathscr{C}_{\text {PDR }}$ at larger $A_{\mathrm{V}}$ so that it predicts cold dust emission and matches the Herschel/SPIRE observations (as discussed in Sect. 7.1.3). The resulting SED is shown in Fig. A.1.

\section{References}

Abel, N. P., Ferland, G. J., Shaw, G., \& van Hoof, P. A. M. 2005, ApJS, 161, 65 Abel, N. P., van Hoof, P. A. M., Shaw, G., Ferland, G. J., \& Elwert, T. 2008, ApJ, 686,1125

Abel, N. P., Dudley, C., Fischer, J., Satyapal, S., \& van Hoof, P. A. M. 2009, ApJ, 701, 1147

Adamo, A., Östlin, G., Zackrisson, E., et al. 2010, MNRAS, 407, 870 Baldwin, J. A., Ferland, G. J., Martin, P. G., et al. 1991, ApJ, 374, 580 Beck, S. C., Turner, J. L., \& Kovo, O. 2000, AJ, 120, 244

Bendo, G. J., Galliano, F., \& Madden, S. C. 2012, MNRAS, 423, 197 Bergvall, N., \& Östlin, G. 2002, A\&A, 390, 891

Bergvall, N., Masegosa, J., Östlin, G., \& Cernicharo, J. 2000, A\&A, 359, 41

Bergvall, N., Zackrisson, E., Andersson, B.-G., et al. 2006, A\&A, 448, 513

Boselli, A., Gavazzi, G., Lequeux, J., \& Pierini, D. 2002, A\&A, 385, 454

Boyer, M. L., Srinivasan, S., Riebel, D., et al. 2012, ApJ, 748, 40

Brauher, J. R., Dale, D. A., \& Helou, G. 2008, ApJS, 178, 280

Calzetti, D., Armus, L., Bohlin, R. C., et al. 2000, ApJ, 533, 682

Calzetti, D., Kennicutt, Jr., R. C., Bianchi, L., et al. 2005, ApJ, 633, 871

Carral, P., Hollenbach, D. J., Lord, S. D., et al. 1994, ApJ, 423, 223

Chyży, K. T., Weżgowiec, M., Beck, R., \& Bomans, D. J. 2011, A\&A, 529, A94

Contursi, A., et al. 2012, A\&A, accepted [arXiv: 1210. 3496]

Cormier, D., Madden, S. C., Hony, S., et al. 2010, A\&A, 518, L57

Croxall, K. V., Smith, J. D., Wolfire, M. G., et al. 2012, ApJ, 747, 81

Crutcher, R. M. 1999, ApJ, 520, 706

de Looze, I., Baes, M., Bendo, G. J., Cortese, L., \& Fritz, J. 2011, MNRAS, 416, 2712

Dopita, M. A., Groves, B. A., Sutherland, R. S., \& Kewley, L. J. 2003, ApJ, 583, 727

Dopita, M. A., Groves, B. A., Fischera, J., et al. 2005, ApJ, 619, 755

Dopita, M. A., Armus, L., Kewley, L. J., et al. 2011, Ap\&SS, 333, 225
Draine, B. T., \& Li, A. 2001, ApJ, 551, 807

Ferland, G. J., Korista, K. T., Verner, D. A., et al. 1998, PASP, 110, 761 Galametz, M., Madden, S., Galliano, F., et al. 2009, A\&A, 508, 645 Galametz, M., Madden, S. C., Galliano, F., et al. 2010, A\&A, 518, L55 Galametz, M., Madden, S. C., Galliano, F., et al. 2011, A\&A, 532, A56 Galliano, F., Madden, S. C., Jones, A. P., et al. 2003, A\&A, 407, 159

Galliano, F., Madden, S. C., Jones, A. P., Wilson, C. D., \& Bernard, J.-P. 2005, A\&A, 434, 867

Galliano, F., Hony, S., Bernard, J.-P., et al. 2011, A\&A, 536, A88

Giveon, U., Sternberg, A., Lutz, D., Feuchtgruber, H., \& Pauldrach, A. W. A. 2002, ApJ, 566, 880

Graciá-Carpio, J., Sturm, E., Hailey-Dunsheath, S., et al. 2011, ApJ, 728, L7

Griffin, M. J., Abergel, A., Abreu, A., et al. 2010, A\&A, 518, L3

Grimes, J. P., Heckman, T., Strickland, D., et al. 2007, ApJ, 668, 891

Grimes, J. P., Heckman, T., Aloisi, A., et al. 2009, ApJS, 181, 272

Groves, B., Dopita, M. A., Sutherland, R. S., et al. 2008, ApJS, 176, 438

Guseva, N. G., Izotov, Y. I., Fricke, K. J., \& Henkel, C. 2012, A\&A, 541, A115

Hayes, M., Östlin, G., Atek, H., et al. 2007, MNRAS, 382, 1465

Heiles, C. 1994, ApJ, 436, 720

Helou, G., Khan, I. R., Malek, L., \& Boehmer, L. 1988, ApJS, 68, 151

Higdon, S. J. U., Devost, D., Higdon, J. L., et al. 2004, PASP, 116, 975

Houck, J. R., Shure, M. A., Gull, G. E., \& Herter, T. 1984, ApJ, 287, L11

Houck, J. R., Roellig, T. L., Van Cleve, J., et al. 2004, in SPIE Conf. Ser., 5487, ed. J. C. Mather, 62

Hunter, D. A., Kaufman, M., Hollenbach, D. J., et al. 2001, ApJ, 553, 121

Iglesias-Páramo, J., Buat, V., Takeuchi, T. T., et al. 2006, ApJS, 164, 38

Izotov, Y. I., \& Thuan, T. X. 1998, ApJ, 500, 188

Izotov, Y. I., \& Thuan, T. X. 1999, ApJ, 511, 639

Jarrett, T. H., Chester, T., Cutri, R., et al. 2000, AJ, 119, 2498

Johnson, K. E., \& Kobulnicky, H. A. 2003, ApJ, 597, 923

Jones, A. P., Tielens, A. G. G. M., \& Hollenbach, D. J. 1996, ApJ, 469, 740

Kaufman, M. J., Wolfire, M. G., Hollenbach, D. J., \& Luhman, M. L. 1999, ApJ, 527, 795

Kaufman, M. J., Wolfire, M. G., \& Hollenbach, D. J. 2006, ApJ, 644, 283

Kepley, A. A., Mühle, S., Everett, J., et al. 2010, ApJ, 712, 536

Kepley, A. A., Zweibel, E. G., Wilcots, E. M., Johnson, K. E., \& Robishaw, T. 2011, ApJ, 736, 139

Kingsburgh, R. L., \& Barlow, M. J. 1992, MNRAS, 257, 317

Kingsburgh, R. L., \& Barlow, M. J. 1994, MNRAS, 271, 257

Kunth, D., \& Östlin, G. 2000, A\&ARv, 10, 1

Kunth, D., Mas-Hesse, J. M., Terlevich, E., et al. 1998, A\&A, 334, 11 
Kunth, D., Leitherer, C., Mas-Hesse, J. M., Östlin, G., \& Petrosian, A. 2003, ApJ, 597, 263

Lauberts, A., \& Valentijn, E. A. 1989, The surface photometry catalogue of the ESO-Uppsala galaxies

Lebouteiller, V., Bernard-Salas, J., Sloan, G. C., \& Barry, D. J. 2010, PASP, 122, 231

Lebouteiller, V., Barry, D. J., Spoon, H. W. W., et al. 2011, ApJS, 196, 8

Lebouteiller, V., et al. 2012, A\&A, submitted

Leitet, E., Bergvall, N., Piskunov, N., \& Andersson, B.-G. 2011, A\&A, 532, A107

Leitherer, C., Ortiz Otálvaro, P. A., Bresolin, F., et al. 2010, ApJS, 189, 309

Leroy, A. K., Bolatto, A., Gordon, K., et al. 2011, ApJ, 737, 12

Lonsdale, C. J., Helou, G., Good, J. C., \& Rice, W. L. 1985

Lutz, D., Kunze, D., Spoon, H. W. W., \& Thornley, M. D. 1998, A\&A, 333, L75

Madden, S. C. 2000, New Astron. Rev., 44, 249

Madden, S. C. 2008, in EAS Pub. Ser. 31, eds. C. Kramer, S. Aalto, \& R. Simon, 123

Madden, S. C., Geis, N., Genzel, R., et al. 1993, ApJ, 407, 579

Madden, S. C., Geis, N., Townes, C. H., et al. 1995, in From Gas to Stars to Dust, eds. M. R. Haas, J. A. Davidson, \& E. F. Erickson, ASP Conf. Ser., 73, 181

Madden, S. C., Galliano, F., Jones, A. P., \& Sauvage, M. 2006, A\&A, 446, 877

Madden, S. C., Remy, A., Galliano, F., et al. 2012, in The Spectral Energy Distribution of Galaxies, Proceeding of the International Astronomical Union, IAU Symp., 284, 141

Maiolino, R., Caselli, P., Nagao, T., et al. 2009, A\&A, 500, L1

Malhotra, S., Kaufman, M. J., Hollenbach, D., et al. 2001, ApJ, 561, 766

Mathis, J. S., Rumpl, W., \& Nordsieck, K. H. 1977, ApJ, 217, 425

Matsuura, M., Barlow, M. J., Zijlstra, A. A., et al. 2009, MNRAS, 396, 918

Micheva, G., Zackrisson, E., Östlin, G., Bergvall, N., \& Pursimo, T. 2010, MNRAS, 405, 1203

Narayanan, D., Krumholz, M. R., Ostriker, E. C., \& Hernquist, L. 2012, MNRAS, 421, 3127

Negishi, T., Onaka, T., Chan, K., \& Roellig, T. L. 2001, A\&A, 375, 566

Oberst, T. E., Parshley, S. C., Stacey, G. J., et al. 2006, ApJ, 652, L125

O'Halloran, B., Madden, S. C., \& Abel, N. P. 2008, ApJ, 681, 1205

O'Halloran, B., Galametz, M., Madden, S. C., et al. 2010, A\&A, 518, L58

Osterbrock, D. E., Tran, H. D., \& Veilleux, S. 1992, ApJ, 389, 305

Östlin, G., Amram, P., Masegosa, J., Bergvall, N., \& Boulesteix, J. 1999, A\&AS, 137,419

Östlin, G., Amram, P., Bergvall, N., et al. 2001, A\&A, 374, 800
Ott, S. 2010, in Astronomical Data Analysis Software and Systems XIX, eds. Y. Mizumoto, K.-I. Morita, \& M. Ohishi, ASP Conf. Ser., 434, 139

Overzier, R. A., Heckman, T. M., Kauffmann, G., et al. 2008, ApJ, 677, 37

Pierini, D., Leech, K. J., \& Völk, H. J. 2003, A\&A, 397, 871

Pilbratt, G. L., Riedinger, J. R., Passvogel, T., et al. 2010, A\&A, 518, L1

Poglitsch, A., Krabbe, A., Madden, S. C., et al. 1995, ApJ, 454, 293

Poglitsch, A., Waelkens, C., Geis, N., et al. 2010, A\&A, 518, L2

Pustilnik, S., Kniazev, A., Pramskij, A., et al. 2004, A\&A, 419, 469

Robishaw, T., Quataert, E., \& Heiles, C. 2008, ApJ, 680, 981

Röllig, M., Ossenkopf, V., Jeyakumar, S., Stutzki, J., \& Sternberg, A. 2006, A\&A, 451, 917

Rubin, R. H., Simpson, J. P., Haas, M. R., \& Erickson, E. F. 1991, ApJ, 374, 564

Rubin, R. H., Dufour, R. J., \& Walter, D. K. 1993, ApJ, 413, 242

Rubin, R. H., Simpson, J. P., Lord, S. D., et al. 1994, ApJ, 420, 772

Sanders, D. B., Mazzarella, J. M., Kim, D., Surace, J. A., \& Soifer, B. T. 2003a, AJ, 126, 1607

Sanders, D. B., Mazzarella, J. M., Kim, D.-C., Surace, J. A., \& Soifer, B. T. 2003b, AJ, 126, 1607

Schaerer, D., \& Stasińska, G. 1999, A\&A, 345, L17

Schruba, A., Leroy, A. K., Walter, F., et al. 2012, AJ, 143, 138

Shaw, G., Ferland, G. J., Henney, W. J., et al. 2009, ApJ, 701, 677

Siebenmorgen, R., \& Krügel, E. 2007, A\&A, 461, 445

Spinoglio, L., Dasyra, K. M., Franceschini, A., et al. 2012, ApJ, 745, 171

Stacey, G. J., Geis, N., Genzel, R., et al. 1991, ApJ, 373, 423

Stacey, G. J., Hailey-Dunsheath, S., Ferkinhoff, C., et al. 2010, ApJ, 724, 957

Strong, A. W., Bloemen, J. B. G. M., Dame, T. M., et al. 1988, A\&A, 207, 1

Tajer, M., Trinchieri, G., Wolter, A., et al. 2005, A\&A, 435, 799

Thornley, M. D., Schreiber, N. M. F., Lutz, D., et al. 2000, ApJ, 539, 641

Thuan, T. X., Izotov, Y. I., \& Lipovetsky, V. A. 1996, ApJ, 463, 120

Tielens, A. G. G. M., \& Hollenbach, D. 1985, ApJ, 291, 722

Turner, J. L., Beck, S. C., \& Ho, P. T. P. 2000, ApJ, 532, L109

Vader, J. P., Frogel, J. A., Terndrup, D. M., \& Heisler, C. A. 1993, AJ, 106, 1743

Vasta, M., Barlow, M. J., Viti, S., Yates, J. A., \& Bell, T. A. 2010, MNRAS, 404, 1910

Werner, M. W., Roellig, T. L., Low, F. J., et al. 2004, ApJS, 154, 1

Wolfire, M. G., Tielens, A. G. G. M., \& Hollenbach, D. 1990, ApJ, 358, 116

Wolfire, M. G., Hollenbach, D., \& McKee, C. F. 2010, ApJ, 716, 1191

Wu, Y., Charmandaris, V., Hao, L., et al. 2006, ApJ, 639, 157

Wu, Y., Bernard-Salas, J., Charmandaris, V., et al. 2008, ApJ, 673, 193

Zubko, V., Dwek, E., \& Arendt, R. G. 2004, ApJS, 152, 211 